\title{
Stochastic Differential Games and Viscosity Solutions of Hamilton-Jacobi-Bellman-Isaacs Equations *
}

\author{
Rainer Buckdahn \\ Département de Mathématiques, Université de Bretagne Occidentale, \\ 6, avenue Victor-le-Gorgeu, B.P. 809, 29285 Brest cedex, France. \\ E-mail:Rainer.Buckdahn@univ-brest.fr. \\ Juan Li \\ School of Mathematical Sciences, Fudan University, Shanghai 200433, \\ Department of Mathematics, Shandong University at Weihai, Weihai 264200, P. R. China. \\ E-mail: juanli@sdu.edu.cn.
}

\begin{abstract}
In this paper we study zero-sum two-player stochastic differential games with the help of theory of Backward Stochastic Differential Equations (BSDEs). At the one hand we generalize the results of the pioneer work of Fleming and Souganidis [8] by considering cost functionals defined by controlled BSDEs and by allowing the admissible control processes to depend on events occurring before the beginning of the game (which implies that the cost functionals become random variables), on the other hand the application of BSDE methods, in particular that of the notion of stochastic "backward semigroups" introduced by Peng [14] allows to prove a dynamic programming principle for the upper and the lower value functions of the game in a straight-forward way, without passing by additional approximations. The upper and the lower value functions are proved to be the unique viscosity solutions of the upper and the lower Hamilton-Jacobi-Bellman-Isaacs equations, respectively. For this Peng's BSDE method (Peng [14]) is translated from the framework of stochastic control theory into that of stochastic differential games.
\end{abstract}

AMS Subject classification: 93E05, 90C39

Keywords: Stochastic Differential Games; Value Function; Backward Stochastic Differential Equations; Dynamic Programming Principle; Viscosity Solution

\footnotetext{
*The work of Mme LI has been supported by a one-year fellowship awarded by the General Council of Finistère, France, the NSF of P.R.China (No. 10426022; 10371067) and Program for Changjiang Scholars and Innovative Research Team in University (PCSIRT).
} 


\section{Introduction}

With their pioneer paper of 1989 Fleming and Souganidis [8] were the first to study in a rigorous manner two-player zero-sum stochastic differential games and to prove that the lower and the upper value functions of such games satisfy the dynamic programming principle, that they are the unique viscosity solutions of the associated Bellman-Isaacs equations and coincide under the Isaacs condition. Their work has translated former results by Evans and Souganidis [7] from a deterministic into the stochastic framework and has given an important impulse for the research in the theory of stochastic differential games. And so a lot of recent works are based on the ideas developed in [8], see, for instance, Buckdahn, Cardaliaguet, Rainer [4], Hou, Tang [11] and Rainer [16]. The reader interested in this subject is also referred to the references given in [8].

Also the present work investigates two-player zero-sum stochastic differential games, but with two main differences to the setting chosen by Fleming and Souganidis [8] and the other papers mentioned above: At the one hand we allow our admissible control processes to depend on the full past of the trajectories of the driving Brownian motion, this means, in particular they can also depend on information occurring before the beginning of the game (which has the consequence that the cost functionals become random variables), on the other hand we consider a more general running cost functional, which implies that the cost functionals will be given by a backward stochastic differential equation (for short, BSDE). These both extensions of the framework in [8] are crucial because they allow to harmonize the setting for stochastic differential games with that for the stochastic control theory and to simplify considerably the approach in [8] by using BSDE methods.

BSDEs in their general non-linear form were introduced by Pardoux and Peng [12] in 1990. They have been studied since then by a lot of authors and have found various applications, namely in stochastic control, finance and the second order PDE theory. BSDE methods, originally developed by Peng [14], [15] for the stochastic control theory, have been introduced in the theory of stochastic differential games by Hamadène, Lepeltier [9] and Hamadène, Lepeltier and Peng [10] to study games with a dynamics whose diffusion coefficient is strictly elliptic and doesn't depend on the controls. In our present work there isn't any such restriction on the diffusion coefficient and the application of BSDE methods, in particular the notion of stochastic backward semigroups (Peng [14]), allows to prove the dynamic programming principle for the upper and lower value functions of the game in a very straight-forward way (i.e., in particular without making use of $r$-strategies and $\pi$-admissible strategies playing an essential role in [8]) and to derive from it with the help of Peng's method (see [14], [15]) the associated Bellman-Isaacs equations.

The dynamics of the stochastic differential game we investigate is given by the controlled stochastic differential equation

$$
\left\{\begin{array}{rl}
d X_{s}^{t, x ; u, v} & =b\left(s, X_{s}^{t, x ; u, v}, u_{s}, v_{s}\right) d s+\sigma\left(s, X_{s}^{t, x ; u, v}, u_{s}, v_{s}\right) d B_{s}, \\
X_{t}^{t, x ; u, v} & =x\left(\in \mathbb{R}^{n}\right),
\end{array} \quad s \in[t, T],\right.
$$

where $T>0$ is an arbitrarily fixed finite time horizon, $B=\left(B_{s}\right)_{s \in[0, T]}$ is a $d$-dimensional standard Brownian motion, and $u=\left(u_{s}\right)_{s \in[t, T]}, v=\left(v_{s}\right)_{s \in[t, T]}$ are progressively measurable with respect to the Brownian filtration and take their values in some compact metric spaces $U$ and $V$, respectively (we will say that $u \in \mathcal{U}_{t, T}, v \in \mathcal{V}_{t, T}$ ). Precise assumptions on the coefficients $b:[0, T] \times \mathbb{R}^{n} \times U \times V \rightarrow$ $\mathbb{R}^{n}$ and $\sigma:[0, T] \times \mathbb{R}^{n} \times U \times V \rightarrow \mathbb{R}^{n \times d}$ are given in the next section. 
The cost functional (interpreted as a payoff for Player I and as a cost for Player II) is introduced by a backward stochastic differential equation (BSDE, for short):

$$
\left\{\begin{array}{rlr}
-d Y_{s}^{t, x ; u, v} & =f\left(s, X_{s}^{t, x ; u, v}, Y_{s}^{t, x ; u, v}, Z_{s}^{t, x ; u, v}, u_{s}, v_{s}\right) d s-Z_{s}^{t, x ; u, v} d B_{s} \\
Y_{T}^{t, x ; u, v} & =\Phi\left(X_{T}^{t, x ; u, v}\right), & s \in[t, T]
\end{array}\right.
$$

where the driver $f:[0, T] \times \mathbb{R}^{n} \times \mathbb{R} \times \mathbb{R}^{d} \times U \times V \rightarrow \mathbb{R}$ describes the running cost and $\Phi: \mathbb{R}^{n} \rightarrow \mathbb{R}$ the terminal cost. Under the assumptions on $f$ and $\Phi$ that will be introduced in the next section the above BSDE has a unique solution $\left(Y_{s}^{t, x ; u, v}, Z_{s}^{t, x ; u, v}\right)_{s \in[t, T]}$ and the cost functional is given by

$$
J(t, x ; u, v)=Y_{t}^{t, x ; u, v} .
$$

As usual in the differential game theory, the players cannot restrict to play only control processes, one player has to fix a strategy while the other player chooses the best answer to this strategy in form of a control process. A strategy admissible for Player I (resp., Player II) is a non-anticipating mapping $\alpha: \mathcal{V}_{t, T} \rightarrow \mathcal{U}_{t, T}$ (resp., $\beta: \mathcal{U}_{t, T} \rightarrow \mathcal{V}_{t, T}$ ) which associates every admissible control of the other player with one of his own admissible controls (we write: $\alpha \in \mathcal{A}_{t, T}, \beta \in \mathcal{B}_{t, T}$; the precise definitions can be found in Section 4). We define the lower value function of our stochastic differential game as follows:

$$
W(t, x):=\operatorname{essinf}_{\beta \in \mathcal{B}_{t, T}} \operatorname{esssup}_{u \in \mathcal{U}_{t, T}} J(t, x ; u, \beta(u))
$$

and the upper value function is given by

$$
U(t, x):=\operatorname{esssup}_{\alpha \in \mathcal{A}_{t, T}} \operatorname{essinf}_{v \in \mathcal{V}_{t, T}} J(t, x ; \alpha(v), v)
$$

The objective of our paper is to investigate these lower and upper value functions. The main results of the paper state that $W$ and $U$ are deterministic (Proposition 4.1) continuous viscosity solutions of the Bellman-Isaacs equations (Theorem 5.1)

$$
\left\{\begin{array}{l}
\frac{\partial}{\partial t} W(t, x)+H^{-}\left(t, x, W, D W, D^{2} W\right)=0, \quad(t, x) \in[0, T) \times \mathbb{R}^{n}, \\
W(T, x)=\Phi(x), \quad x \in \mathbb{R}^{n},
\end{array}\right.
$$

and

$$
\left\{\begin{array}{l}
\frac{\partial}{\partial t} U(t, x)+H^{+}\left(t, x, U, D U, D^{2} U\right)=0, \quad(t, x) \in[0, T) \times \mathbb{R}^{n}, \\
U(T, x)=\Phi(x), \quad x \in \mathbb{R}^{n},
\end{array}\right.
$$

respectively, associated with the Hamiltonians

$$
\begin{aligned}
& H^{-}(t, x, y, p, X)=\sup _{u \in U} \inf _{v \in V} H(t, x, y, p, X, u, v), \\
& H^{+}(t, x, y, p, X)=\inf _{v \in V} \sup _{u \in U} H(t, x, y, p, X, u, v),
\end{aligned}
$$

$(t, x, y, p, X) \in[0, T] \times \mathbb{R}^{n} \times \mathbb{R} \times \mathbb{R}^{n} \times S^{n}$ (Recall that $S^{n}$ denotes the set of all $n \times n$ symmetric matrices), where

$$
\begin{aligned}
H(t, x, y, p, X, u, v) & =1 / 2 \cdot \operatorname{tr}\left(\sigma \sigma^{T}(t, x, u, v) X\right) \\
& +p \cdot b(t, x, u, v)+f(t, x, y, p \cdot \sigma(t, x, u, v), u, v) .
\end{aligned}
$$


Moreover, we prove the uniqueness (Theorem 6.1) in a class of continuous functions with a growth condition which was introduced by Barles, Buckdahn and Pardoux [3] and is weaker than the polynomial growth assumption.

Notice that the fact that $W$ and $U$, introduced as combination of essential infimum and essential supremum over a class of random variables, are deterministic is far from beng trivial. The method developed by Peng $[14,15]$ (see also Theorem 3.1 of the present paper) for value functions involving only control processes but not strategies doesn't apply here since the strategies from $\mathcal{A}_{t, T}$ and $\mathcal{B}_{t, T}$ don't have, in general, any continuity property. To overcome this difficulty we show in Proposition 4.1 and Lemma 4.1 that $W$ and $U$ are invariant under Girsanov transformation and use the fact that a functional of the Brownian motion which is invariant under Girsanov transformation into all directions of the Cameron-Martin space must be deterministic. We emphasize that the proof of Lemma 4.1 doesn't use BSDE methods which makes this method also applicable to the other situations, such as standard stochastic control problems.

Our paper is organized as follows. The Sections 2 and 3 recall some elements of the theory of backward SDEs and forward-backward SDEs which will be needed in the sequel. Section 4 introduces the setting of the stochastic differential game and its lower and upper value functions $W$ and $U$, and proves that these both functions are deterministic and satisfy the dynamic programming principle (for short, DPP). The DPP allows to derive in Section 5 with the help of Peng's method that $W$ and $U$ are viscosity solutions of the associated Bellman-Isaacs equations; the uniqueness is studied in Section 6. Finally, after having characterized $W$ and $U$ as unique viscosity solutions of associated Bellman-Isaacs equations we show that under the Isaacs condition $W$ and $U$ coincide (one says that the game has a value) and we also identify $W$ and $U$ with the value functions defined in $[8]$.

\section{Preliminaries}

Let us begin by introducing the setting for the stochastic differential game we want to investigate. We consider as Brownian motion B is the d-dimensional coordinate process on the classical Wiener space $(\Omega, \mathcal{F}, P)$, i.e., $\Omega$ is the set of continuous functions from $[0, \mathrm{~T}]$ to $\mathbb{R}^{d}$ starting from $0\left(\Omega=C_{0}\left([0, T] ; \mathbb{R}^{d}\right)\right), \mathcal{F}$ the completed Borel $\sigma$-algebra over $\Omega, \mathrm{P}$ the Wiener measure and $\mathrm{B}$ the canonical process: $B_{s}(\omega)=\omega_{s}, s \in[0, T], \omega \in \Omega$. By $\left\{\mathcal{F}_{s}, 0 \leq s \leq T\right\}$ we denote the natural filtration generated by $\left\{B_{s}\right\}_{0 \leq s \leq T}$ and augmented by all P-null sets, i.e.,

$$
\mathcal{F}_{s}=\sigma\left\{B_{r}, r \leq s\right\} \vee \mathcal{N}_{P}, \quad s \in[0, T],
$$

where $\mathcal{N}_{P}$ is the set of all P-null subsets, and $T>0$ a fixed real time horizon. For any $n \geq 1$, $|z|$ denotes the Euclidean norm of $z \in \mathbb{R}^{n}$. We also shall introduce the following both spaces of processes which will be used frequently in the sequel:

$$
\begin{gathered}
\mathcal{S}^{2}(0, T ; \mathbb{R}):=\left\{\left(\psi_{t}\right)_{0 \leq t \leq T}\right. \text { real-valued adapted càdlàg process : } \\
\left.E\left[\sup _{0 \leq t \leq T}\left|\psi_{t}\right|^{2}\right]<+\infty\right\} ; \\
\mathcal{H}^{2}\left(0, T ; \mathbb{R}^{n}\right):=\left\{\left(\psi_{t}\right)_{0 \leq t \leq T} \mathbb{R}^{n}\right. \text {-valued progressively measurable process : } \\
\left.\|\psi\|_{2}^{2}=E\left[\int_{0}^{T}\left|\psi_{t}\right|^{2} d t\right]<+\infty\right\} .
\end{gathered}
$$


Let us now consider a function $g: \Omega \times[0, T] \times \mathbb{R} \times \mathbb{R}^{d} \rightarrow \mathbb{R}$ with the property that $(g(t, y, z))_{t \in[0, T]}$ is progressively measurable for each $(y, z)$ in $\mathbb{R} \times \mathbb{R}^{d}$, and we also make the following assumptions on $g$ throughout the paper:

(A1) There exists a constant $C \geq 0$ such that, P-a.s., for all $t \in[0, T], y_{1}, y_{2} \in \mathbb{R}, z_{1}, z_{2} \in \mathbb{R}^{d}$,

$$
\left|g\left(t, y_{1}, z_{1}\right)-g\left(t, y_{2}, z_{2}\right)\right| \leq C\left(\left|y_{1}-y_{2}\right|+\left|z_{1}-z_{2}\right|\right) .
$$

(A2) $g(\cdot, 0,0) \in \mathcal{H}^{2}(0, T ; \mathbb{R})$.

The following result on backward stochastic differential equations (BSDEs) is by now well known, for its proof the reader is referred to Pardoux and Peng [12].

Lemma 2.1. Under the assumptions (A1) and (A2), for any random variable $\xi \in L^{2}\left(\varnothing, \mathcal{F}_{T}, P\right)$, the BSDE

$$
y_{t}=\xi+\int_{t}^{T} g\left(s, y_{s}, z_{s}\right) d s-\int_{t}^{T} z_{s} d B_{s}, \quad 0 \leq t \leq T,
$$

has a unique adapted solution

$$
\left(y_{t}^{T, g, \xi}, z_{t}^{T, g, \xi}\right)_{t \in[0, T]} \in \mathcal{S}^{2}(0, T ; \mathbb{R}) \times \mathcal{H}^{2}\left(0, T ; \mathbb{R}^{d}\right) .
$$

In the sequel, we always assume that the driving coefficient $g$ of a BSDE satisfies (A1) and (A2).

Let us remark that Lemma 2.1 remains true when assumption (A1) is replaced by weaker assumptions, for instance those studied in Bahlali [1], Bahlali, Essaky, Hassani and Pardoux [2] or Pardoux and Peng [13. However, here, for the sake of simplicity of the calculus we prefer to work with the Lipschitz assumption.

We also shall recall the following both basic results on BSDEs. We begin with the well-known comparison theorem (see El Karoui, Peng, Quenez [6]).

Lemma 2.2. (Comparison Theorem) Given two coefficients $g_{1}$ and $g_{2}$ satisfying (A1) and (A2) and two terminal values $\xi_{1}, \xi_{2} \in L^{2}\left(\Omega, \mathcal{F}_{T}, P\right)$, we denote by $\left(y^{1}, z^{1}\right)$ and $\left(y^{2}, z^{2}\right)$ the solution of BSDE with the data $\left(\xi_{1}, g_{1}\right)$ and $\left(\xi_{2}, g_{2}\right)$, respectively. Then we have:

(i) (Monotonicity) If $\xi_{1} \geq \xi_{2}$ and $g_{1} \geq g_{2}$, a.s., then $y_{t}^{1} \geq y_{t}^{2}$, a.s., for all $t \in[0, T]$.

(ii) (Strict Monotonicity) If, in addition to (i), we also assume that $P\left(\xi_{1}>\xi_{2}\right)>0$, then $P\left\{y_{t}^{1}>y_{t}^{2}\right\}>0,0 \leq t \leq T$, and in particular, $y_{0}^{1}>y_{0}^{2}$.

Using the notation introduced in Lemma 2.2 we now suppose that, for some $g: \Omega \times[0, T] \times$ $\mathbb{R} \times \mathbb{R}^{d} \longrightarrow \mathbb{R}$ satisfying (A1) and (A2) and for some $i \in\{1,2\}$, the drivers $g_{i}, i=1,2$, are of the form

$$
g_{i}\left(s, y_{s}^{i}, z_{s}^{i}\right)=g\left(s, y_{s}^{i}, z_{s}^{i}\right)+\varphi_{i}(s), \text { dsdP-a.e., } i=1,2,
$$

where $\varphi_{i} \in \mathcal{H}^{2}(0, T ; \mathbb{R}), \quad i=1,2$. Then, for terminal values $\xi_{1}, \xi_{2}$ belonging to $L^{2}\left(\Omega, \mathcal{F}_{T}, P\right)$ we have the following

Lemma 2.3. The difference of the solutions $\left(y^{1}, z^{1}\right)$ and $\left(y^{2}, z^{2}\right)$ of BSDE with the data $\left(\xi_{1}, g_{1}\right)$ and $\left(\xi_{2}, g_{2}\right)$, respectively, satisfies the following estimate:

$$
\begin{aligned}
& \left|y_{t}^{1}-y_{t}^{2}\right|^{2}+\frac{1}{2} E\left[\int_{t}^{T} e^{\beta(s-t)}\left[\left|y_{s}^{1}-y_{s}^{2}\right|^{2}+\left|z_{s}^{1}-z_{s}^{2}\right|^{2}\right] d s \mid \mathcal{F}_{t}\right] \\
\leq & E\left[e^{\beta(T-t)}\left|\xi_{1}-\xi_{2}\right|^{2} \mid \mathcal{F}_{t}\right]+E\left[\int_{t}^{T} e^{\beta(s-t)}\left|\varphi_{1}(s)-\varphi_{2}(s)\right|^{2} d s \mid \mathcal{F}_{t}\right], \quad P \text {-a.s., for all } 0 \leq t \leq T,
\end{aligned}
$$

where $\beta=16\left(1+C^{2}\right)$.

For the proof the reader is referred to El Karoui, Peng, Quenez [6] or Peng [14]. 


\section{Forward- Backward SDES (FBSDEs)}

In this section we give an overview over basic results on BSDEs associated with Forward SDEs (for short: FSDEs). We consider measurable functions $b:[0, T] \times \Omega \times \mathbb{R}^{n} \rightarrow \mathbb{R}^{n}$ and $\sigma:[0, T] \times \Omega \times \mathbb{R}^{n} \rightarrow \mathbb{R}^{n \times d}$ which are supposed to satisfy the following conditions:

(i) $\quad b(\cdot, 0)$ and $\sigma(\cdot, 0)$ are $\mathcal{F}_{t}$ - adapted processes, and there exists some constant $C>0$ such that

$$
|b(t, x)|+|\sigma(t, x)| \leq C(1+|x|) \text {,a.s., for all } 0 \leq t \leq T, x \in \mathbb{R}^{n} ;
$$

(ii) $b$ and $\sigma$ are Lipschitz in $x$, i.e., there is some constant $C>0$ such that

$$
\begin{aligned}
\left|b(t, x)-b\left(t, x^{\prime}\right)\right|+\left|\sigma(t, x)-\sigma\left(t, x^{\prime}\right)\right| & \leq C\left|x-x^{\prime}\right|, \text { a.s. }, \\
& \text { for all } 0 \leq t \leq T, x, x^{\prime} \in \mathbb{R}^{n} .
\end{aligned}
$$

We now consider the following SDE parameterized by the initial condition $(t, \zeta) \in[0, T] \times$ $L^{2}\left(\Omega, \mathcal{F}_{t}, P ; \mathbb{R}^{n}\right):$

$$
\left\{\begin{aligned}
d X_{s}^{t, \zeta} & =b\left(s, X_{s}^{t, \zeta}\right) d s+\sigma\left(s, X_{s}^{t, \zeta}\right) d B_{s}, s \in[t, T], \\
X_{t}^{t, \zeta} & =\zeta .
\end{aligned}\right.
$$

Under the assumption (H3.1), SDE (3.1) has a unique strong solution and, for any $p \geq 2$, there exists $C_{p} \in \mathbb{R}$ such that, for any $t \in[0, T]$ and $\zeta, \zeta^{\prime} \in L^{p}\left(\Omega, \mathcal{F}_{t}, P ; \mathbb{R}^{n}\right)$,

$$
\begin{aligned}
E\left[\sup _{t \leq s \leq T}\left|X_{s}^{t, \zeta}-X_{s}^{t, \zeta^{\prime}}\right|^{p} \mid \mathcal{F}_{t}\right] & \leq C_{p}\left|\zeta-\zeta^{\prime}\right|^{p}, \quad \text { a.s. } \\
E\left[\sup _{t \leq s \leq T}\left|X_{s}^{t, \zeta}\right|^{p} \mid \mathcal{F}_{t}\right] & \leq C_{p}\left(1+|\zeta|^{p}\right), \quad \text { a.s.. }
\end{aligned}
$$

We emphasize that the constant $C_{p}$ in (3.2) only depends on the Lipschitz and the growth constants of $b$ and $\sigma$. Let now be given two real valued functions $f(t, x, y, z)$ and $\Phi(x)$ which shall satisfy the following conditions:

(i) $\quad \Phi: \Omega \times \mathbb{R}^{n} \rightarrow \mathbb{R}$ is an $\mathcal{F}_{T} \otimes \mathcal{B}\left(\mathbb{R}^{n}\right)$-measurable random variable and $f:[0, T] \times \Omega \times \mathbb{R}^{n} \times \mathbb{R} \times \mathbb{R}^{d} \rightarrow \mathbb{R}$ is a measurable process such that

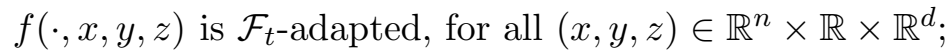

(ii) There exists a constant $C>0$ such that

$$
\begin{aligned}
&\left|f(t, x, y, z)-f\left(t, x^{\prime}, y^{\prime}, z^{\prime}\right)\right|+\left|\Phi(x)-\Phi\left(x^{\prime}\right)\right| \\
& \leq C\left(\left|x-x^{\prime}\right|+\left|y-y^{\prime}\right|+\left|z-z^{\prime}\right|\right), \quad \text { a.s. } \\
& \text { for all } 0 \leq t \leq T, x, x^{\prime} \in \mathbb{R}^{n}, y, y^{\prime} \in \mathbb{R} \text { and } z, z^{\prime} \in \mathbb{R}^{d}
\end{aligned}
$$

(iii) $\quad f$ and $\Phi$ satisfy a linear growth condition, i.e., there exists some $C>0$ such that, $\mathrm{dt} \times \mathrm{dP}$-a.e., for all $x \in \mathbb{R}^{n}$,

$$
|f(t, x, 0,0)|+|\Phi(x)| \leq C(1+|x|) .
$$

With the help of the above assumptions we can verify that the coefficient $f\left(s, X_{s}^{t, \zeta}, y, z\right)$ satisfies the hypotheses (A1), (A2) and $\xi=\Phi\left(X_{T}^{t, \zeta}\right) \in L^{2}\left(\Omega, \mathcal{F}_{T}, P ; \mathbb{R}\right)$. Therefore, the following BSDE possesses a unique solution:

$$
\left\{\begin{aligned}
-d Y_{s}^{t, \zeta} & =f\left(s, X_{s}^{t, \zeta}, Y_{s}^{t, \zeta}, Z_{s}^{t, \zeta}\right) d s-Z_{s}^{t, \zeta} d B_{s}, s \in[t, T], \\
Y_{T}^{t, \zeta} & =\Phi\left(X_{T}^{t, \zeta}\right) .
\end{aligned}\right.
$$


Proposition 3.1. We suppose that the hypotheses (H3.1) and (H3.2) hold. Then, for any $0 \leq t \leq T$ and the associated initial conditions $\zeta, \zeta^{\prime} \in L^{2}\left(\Omega, \mathcal{F}_{t}, P ; \mathbb{R}^{n}\right)$, we have the following estimates:

In particular,

$$
\begin{aligned}
& \text { (i) } E\left[\sup _{t \leq s \leq T}\left|Y_{s}^{t, \zeta}\right|^{2}+\int_{t}^{T}\left|Z_{s}^{t, \zeta}\right|^{2} d s \mid \mathcal{F}_{t}\right] \leq C\left(1+|\zeta|^{2}\right) \text {, a.s.; } \\
& \text { (ii) } E\left[\sup _{t \leq s \leq T}\left|Y_{s}^{t, \zeta}-Y_{s}^{t, \zeta^{\prime}}\right|^{2}+\int_{t}^{T}\left|Z_{s}^{t, \zeta}-Z_{s}^{t, \zeta^{\prime}}\right|^{2} d s \mid \mathcal{F}_{t}\right] \leq C\left|\zeta-\zeta^{\prime}\right|^{2} \text {, a.s.. }
\end{aligned}
$$

$$
\begin{aligned}
& \text { (iii) }\left|Y_{t}^{t, \zeta}\right| \leq C(1+|\zeta|), \text { a.s.; } \\
& \text { (iv) }\left|Y_{t}^{t, \zeta}-Y_{t}^{t, \zeta^{\prime}}\right| \leq C\left|\zeta-\zeta^{\prime}\right| \text {, a.s., }
\end{aligned}
$$

where the constant $C>0$ depends only on the Lipschitz and the growth constants of $b, \sigma, f$ and $\Phi$. The proof can be found in Peng [14].

Let us now introduce the random field:

$$
u(t, x)=\left.Y_{s}^{t, x}\right|_{s=t}, \quad(t, x) \in[0, T] \times \mathbb{R}^{n},
$$

where $Y^{t, x}$ is the solution of $\operatorname{BSDE}(3.3)$ with $x \in \mathbb{R}^{n}$ at the place of $\zeta \in L^{2}\left(\Omega, \mathcal{F}_{t}, P ; \mathbb{R}^{n}\right)$.

As a consequence of Proposition 3.1 we have that, for all $t \in[0, T]$, P-a.s.,

$$
\begin{aligned}
& \text { (i) }|u(t, x)-u(t, y)| \leq C|x-y| \text {, for all } x, y \in \mathbb{R}^{n} \text {; } \\
& \text { (ii) }|u(t, x)| \leq C(1+|x|) \text {, for all } x \in \mathbb{R}^{n} \text {. }
\end{aligned}
$$

Remark 3.1. In the general situation $u$ is an adapted random function, that is, for any $x \in$ $\mathbb{R}^{n}, u(\cdot, x)$ is an $\mathcal{F}_{t}$-adapted real valued process. Indeed, recall that $b, \sigma, f$ and $\Phi$ all are $\mathcal{F}_{t}$-adapted random functions. On the other hand, it is well known that, under the additional assumption that the functions

$$
b, \sigma, f \text { and } \Phi \text { are deterministic, }
$$

also $u$ is a deterministic function of $(t, x)$. theorem.

The random field $u$ and $Y^{t, \zeta},(t, \zeta) \in[0, T] \times L^{2}\left(\Omega, \mathcal{F}_{t}, P ; \mathbb{R}^{n}\right)$, are related by the following

Theorem 3.1. Under the assumptions (H3.1) and (H3.2), for any $t \in[0, T]$ and $\zeta \in L^{2}\left(\Omega, \mathcal{F}_{t}, P ; \mathbb{R}^{n}\right)$, we have

$$
u(t, \zeta)=Y_{t}^{t, \zeta}, \quad P-a . s . .
$$

The proof of Theorem 3.1 can be found in Peng [14], we give it for the reader's convenience. It makes use of the following definition.

Definition 3.1. For any $t \in[0, T]$, a sequence $\left\{A_{i}\right\}_{i=1}^{N} \subset \mathcal{F}_{t}$ (with $1 \leq N \leq \infty$ ) is called a partition of $\left(\Omega, \mathcal{F}_{t}\right)$ if $\cup_{i=1}^{N} A_{i}=\Omega$ and $A_{i} \cap A_{j}=\phi$, whenever $i \neq j$.

Proof (of Theorem 3.1): We first consider the case where $\zeta$ is a simple random variable of the form

$$
\zeta=\sum_{i=1}^{N} x_{i} \mathbf{1}_{A_{i}}
$$


where $\left\{A_{i}\right\}_{i=1}^{N}$ is a finite partition of $\left(\Omega, \mathcal{F}_{t}\right)$ and $x_{i} \in \mathbb{R}^{n}$, for $1 \leq i \leq N$.

For each $i$, we put $\left(X_{s}^{i}, Y_{s}^{i}, Z_{s}^{i}\right) \equiv\left(X_{s}^{t, x_{i}}, Y_{s}^{t, x_{i}}, Z_{s}^{t, x_{i}}\right)$. Then $X^{i}$ is the solution of the SDE

$$
X_{s}^{i}=x_{i}+\int_{t}^{s} b\left(r, X_{r}^{i}\right) d r+\int_{t}^{s} \sigma\left(r, X_{r}^{i}\right) d B_{r}, s \in[t, T]
$$

and $\left(Y^{i}, Z^{i}\right)$ is the solution of the associated BSDE

$$
Y_{s}^{i}=\Phi\left(X_{T}^{i}\right)+\int_{s}^{T} f\left(r, X_{r}^{i}, Y_{r}^{i}, Z_{r}^{i}\right) d r-\int_{s}^{T} Z_{r}^{i} d B_{r}, s \in[t, T] .
$$

The above two equations are multiplied by $\mathbf{1}_{A_{i}}$ and summed up with respect to $i$. Thus, taking into account that $\sum_{i} \varphi\left(x_{i}\right) \mathbf{1}_{A_{i}}=\varphi\left(\sum_{i} x_{i} \mathbf{1}_{A_{i}}\right)$, we get

$$
\sum_{i=1}^{N} \mathbf{1}_{A_{i}} X_{s}^{i}=\sum_{i=1}^{N} x_{i} \mathbf{1}_{A_{i}}+\int_{t}^{s} b\left(r, \sum_{i=1}^{N} \mathbf{1}_{A_{i}} X_{r}^{i}\right) d r+\int_{t}^{s} \sigma\left(r, \sum_{i=1}^{N} \mathbf{1}_{A_{i}} X_{r}^{i}\right) d B_{r}
$$

and

$$
\begin{aligned}
\sum_{i=1}^{N} \mathbf{1}_{A_{i}} Y_{s}^{i}= & \Phi\left(\sum_{i=1}^{N} \mathbf{1}_{A_{i}} X_{T}^{i}\right)+\int_{s}^{T} f\left(r, \sum_{i=1}^{N} \mathbf{1}_{A_{i}} X_{r}^{i}, \sum_{i=1}^{N} \mathbf{1}_{A_{i}} Y_{r}^{i}, \sum_{i=1}^{N} \mathbf{1}_{A_{i}} Z_{r}^{i}\right) d r \\
& -\int_{s}^{T} \sum_{i=1}^{N} \mathbf{1}_{A_{i}} Z_{r}^{i} d B_{r} .
\end{aligned}
$$

Then the strong uniqueness property of the solution of the SDE and the BSDE yields

$$
X_{s}^{t, \zeta}=\sum_{i=1}^{N} X_{s}^{i} \mathbf{1}_{A_{i}},\left(Y_{s}^{t, \zeta}, Z_{s}^{t, \zeta}\right)=\left(\sum_{i=1}^{N} \mathbf{1}_{A_{i}} Y_{s}^{i}, \sum_{i=1}^{N} \mathbf{1}_{A_{i}} Z_{s}^{i}\right), s \in[t, T] .
$$

Finally, from $u\left(t, x_{i}\right)=Y_{t}^{i}, 1 \leq i \leq N$, we deduce that

$$
Y_{t}^{t, \zeta}=\sum_{i=1}^{N} Y_{t}^{i} \mathbf{1}_{A_{i}}=\sum_{i=1}^{N} u\left(t, x_{i}\right) \mathbf{1}_{A_{i}}=u\left(t, \sum_{i=1}^{N} x_{i} \mathbf{1}_{A_{i}}\right)=u(t, \zeta) .
$$

Therefore, for simple random variables, we have the desired result.

Given a general $\zeta \in L^{2}\left(\Omega, \mathcal{F}_{t}, P ; \mathbb{R}^{n}\right)$ we can choose a sequence of simple random variables $\left\{\zeta_{i}\right\}$ which converges to $\zeta$ in $L^{2}\left(\Omega, \mathcal{F}_{t}, P ; \mathbb{R}^{n}\right)$. Consequently, from the estimates $(3.4),(3.6)$ and the first step of the proof, we have

$$
\begin{aligned}
E\left|Y_{t}^{t, \zeta_{i}}-Y_{t}^{t, \zeta}\right|^{2} & \leq C E\left|\zeta_{i}-\zeta\right|^{2} \rightarrow 0, i \rightarrow \infty, \\
E\left|u\left(t, \zeta_{i}\right)-u(t, \zeta)\right|^{2} & \leq C E\left|\zeta_{i}-\zeta\right|^{2} \rightarrow 0, i \rightarrow \infty, \\
Y_{t}^{t, \zeta_{i}} & =u\left(t, \zeta_{i}\right), i \geq 1 .
\end{aligned}
$$

Then the proof is complete.

Remark 3.2. Under (H3.1), (H3.2) and (H3.3) we know $u(t, x)$ is $\frac{1}{2}$-Hölder continuous in $t$ : There exists a constant $C$ such that, for every $x \in \mathbb{R}^{n}, t, t^{\prime} \in[0, T]$,

$$
\left|u(t, x)-u\left(t^{\prime}, x\right)\right| \leq C(1+|x|)\left|t-t^{\prime}\right|^{\frac{1}{2}} .
$$

This inequality can be proved with the help of Theorem 3.1. Since, on the other hand, a similar result but in a more general setting will be proved later (see Theorem 4.2) we don't give the proof here.

For the case of random coefficients $b, \sigma, f$ and $\Phi$ we can state the following property. 
Remark 3.3. Let us suppose in addition to the assumptions (H3.1) and (H3.2) that $\sigma(\omega, t, \cdot)$ and $b(\omega, t, \cdot)$ are continuously differentiable with Lipschitz derivative such that, for some constant $C$,

$$
\begin{array}{r}
\left|D_{x} \sigma(\omega, t, x)\right|+\left|D_{x} b(\omega, t, x)\right| \leq C, d t d P \text {-a.e., for all } x \in \mathbb{R}^{n} \\
D_{x} \sigma(\omega, t, \cdot), D_{x} b(\omega, t, \cdot) \text { are Lipschitz, uniformly in }(\omega, t) .
\end{array}
$$

Then the random field $u(\omega, t, x): \Omega \times[0, T] \times \mathbb{R}^{n} \rightarrow \mathbb{R}$ possesses a continuous version.

The proof uses a standard argument based on the properties of the stochastic flow associated with (3.1).

\section{Stochastic Differential Games and Associated Dynamic Programming Prin- ciples}

Now we want to study the stochastic differential game. The set of admissible control processes $\mathcal{U}$ (resp., $\mathcal{V}$ ) for the first (resp., second) player is the set of all $\mathrm{U}$ (resp., V)-valued $\mathcal{F}_{t}$-progressively measurable processes. The control state spaces $U$ and $V$ are supposed to be compact metric spaces.

For given admissible controls $u(\cdot) \in \mathcal{U}$ and $v(\cdot) \in \mathcal{V}$, the according orbit which regards $t$ as the initial time and $\zeta \in L^{2}\left(\Omega, \mathcal{F}_{t}, P ; \mathbb{R}^{n}\right)$ as the initial state is defined by the solution of the following SDE:

$$
\left\{\begin{aligned}
d X_{s}^{t, \zeta ; u, v} & =b\left(s, X_{s}^{t, \zeta ; u, v}, u_{s}, v_{s}\right) d s+\sigma\left(s, X_{s}^{t, \zeta ; u, v}, u_{s}, v_{s}\right) d B_{s}, s \in[t, T], \\
X_{t}^{t, \zeta ; u, v} & =\zeta
\end{aligned}\right.
$$

where the mappings

$$
b:[0, T] \times \mathbb{R}^{n} \times U \times V \rightarrow \mathbb{R}^{n} \text { and } \sigma:[0, T] \times \mathbb{R}^{n} \times U \times V \rightarrow \mathbb{R}^{n \times d}
$$

satisfy the following conditions:

(i) For every fixed $x \in \mathbb{R}^{n}, b(., x, .,$.$) and \sigma(., x, .,$.$) are continuous in (t, u, v)$;

(ii) There exists a $C>0$ such that, for all $t \in[0, T], x, x^{\prime} \in \mathbb{R}^{n}, u \in U, v \in V$,

$$
\left|b(t, x, u, v)-b\left(t, x^{\prime}, u, v\right)\right|+\left|\sigma(t, x, u, v)-\sigma\left(t, x^{\prime}, u, v\right)\right| \leq C\left|x-x^{\prime}\right| .
$$

From (H4.1) we can get the global linear growth conditions of $\mathrm{b}$ and $\sigma$, i.e., the existence of some $C>0$ such that, for all $0 \leq t \leq T, u \in U, v \in V, x \in \mathbb{R}^{n}$,

$$
|b(t, x, u, v)|+|\sigma(t, x, u, v)| \leq C(1+|x|) .
$$

Obviously, under the above assumptions, for any $u(\cdot) \in \mathcal{U}$ and $v(\cdot) \in \mathcal{V}$, SDE (4.1) has a unique strong solution. Moreover, for any $p \geq 2$, there exists $C_{p} \in \mathbb{R}$ such that, for any $t \in[0, T]$, $u(\cdot) \in \mathcal{U}, v(\cdot) \in \mathcal{V}$ and $\zeta, \zeta^{\prime} \in L^{2}\left(\Omega, \mathcal{F}_{t}, P ; \mathbb{R}^{n}\right)$, we also have the following estimates, P-a.s.:

$$
\begin{aligned}
E\left[\sup _{s \in[t, T]}\left|X_{s}^{t, \zeta ; u, v}-X_{s}^{t, \zeta^{\prime} ; u, v}\right| p \mid \mathcal{F}_{t}\right] & \leq C_{p}\left|\zeta-\zeta^{\prime}\right|^{p}, \\
E\left[\sup _{s \in[t, T]}\left|X_{s}^{t, \zeta ; u, v}\right| p \mid \mathcal{F}_{t}\right] & \leq C_{p}\left(1+|\zeta|^{p}\right) .
\end{aligned}
$$

The constant $C_{p}$ depends only on the Lipschitz and the linear growth constants of $b$ and $\sigma$ with respect to $x$. 
Let now be given two functions

$$
\Phi: \mathbb{R}^{n} \rightarrow \mathbb{R}, f:[0, T] \times \mathbb{R}^{n} \times \mathbb{R} \times \mathbb{R}^{d} \times U \times V \rightarrow \mathbb{R}
$$

that satisfy the following conditions:

(i) For every fixed $(x, y, z) \in \mathbb{R}^{n} \times \mathbb{R} \times \mathbb{R}^{d}, f(., x, y, z, . .$.$) is continuous in (t, u, v)$ and there exists a constant $C>0$ such that, for all $t \in[0, T], x, x^{\prime} \in \mathbb{R}^{n}, y, y^{\prime} \in \mathbb{R}, z, z^{\prime}$ $\in \mathbb{R}^{d}, u \in U$ and $v \in V$,

$$
\begin{aligned}
\mid f(t, x, y, z, u, v)- & f\left(t, x^{\prime}, y^{\prime}, z^{\prime}, u, v\right) \mid \\
\leq & C\left(\left|x-x^{\prime}\right|+\left|y-y^{\prime}\right|+\left|z-z^{\prime}\right|\right) ;
\end{aligned}
$$

(ii) There is a constant $C>0$ such that, for all $x, x^{\prime} \in \mathbb{R}^{n}$,

$$
\left|\Phi(x)-\Phi\left(x^{\prime}\right)\right| \leq C\left|x-x^{\prime}\right|
$$

From (H4.2) we see that $f$ and $\Phi$ also satisfy the global linear growth condition in $x$, i.e., there exists some $C>0$ such that, for all $0 \leq t \leq T, u \in U, v \in V, x \in \mathbb{R}^{n}$,

$$
|f(t, x, 0,0, u, v)|+|\Phi(x)| \leq C(1+|x|) .
$$

For any $u(\cdot) \in \mathcal{U}, v(\cdot) \in \mathcal{V}$ and $\zeta \in L^{2}\left(\Omega, \mathcal{F}_{t}, P ; \mathbb{R}^{n}\right)$, the mappings $\xi:=\Phi\left(X_{T}^{t, \zeta ; u, v}\right)$ and $g(s, y, z):=$ $f\left(s, X_{s}^{t, \zeta ; u, v}, y, z, u_{s}, v_{s}\right)$ satisfy the conditions of Lemma 2.1 on the interval $[t, T]$. Therefore, there exists a unique solution to the following BSDE:

$$
\left\{\begin{aligned}
-d Y_{s}^{t, \zeta ; u, v} & =f\left(s, X_{s}^{t, \zeta ; u, v}, Y_{s}^{t, \zeta ; u, v}, Z_{s}^{t, \zeta ; u, v}, u_{s}, v_{s}\right) d s-Z_{s}^{t, \zeta ; u, v} d B_{s}, \\
Y_{T}^{t, \zeta ; u, v} & =\Phi\left(X_{T}^{t, \zeta ; u, v}\right),
\end{aligned}\right.
$$

where $X^{t, \zeta ; u, v}$ is introduced by equation (4.1).

Moreover, in analogy to Proposition 3.1, we can see that there exists some constant $C>0$ such that, for all $0 \leq t \leq T, \zeta, \zeta^{\prime} \in L^{2}\left(\Omega, \mathcal{F}_{t}, P ; \mathbb{R}^{n}\right), u(\cdot) \in \mathcal{U}$ and $v(\cdot) \in \mathcal{V}$, P-a.s.,

$$
\begin{aligned}
& \text { (i) }\left|Y_{t}^{t, \zeta ; u, v}-Y_{t}^{t, \zeta^{\prime} ; u, v}\right| \leq C\left|\zeta-\zeta^{\prime}\right| ; \\
& \text { (ii) }\left|Y_{t}^{t, \zeta ; u, v}\right| \leq C(1+|\zeta|) .
\end{aligned}
$$

We now introduce the following subspaces of admissible controls:

Definition 4.1. An admissible control process $u=\left\{u_{r}, r \in[t, s]\right\}$ (resp., $v=\left\{v_{r}, r \in[t, s]\right\}$ ) for Player I (resp., II) on $[t, s](t<s \leq T)$ is an $\mathcal{F}_{r}$-progressively measurable process taking values in $U$ (resp., $V$ ). The set of all admissible controls for Player I (resp., II) on $[t, s]$ is denoted by $\mathcal{U}_{t, s}\left(\right.$ resp., $\left.\mathcal{V}_{t, s}\right)$. We identify two processes $u$ and $\bar{u}$ in $\mathcal{U}_{t, s}$ and write $u \equiv \bar{u}$ on $[t, s]$, if $P\{u=$ $\bar{u}$ a.e. in $[t, s]\}=1$. Similarly we interpret $v \equiv \bar{v}$ on $[t, s]$ in $\mathcal{V}_{t, s}$.

Finally, we have still to define the admissible strategies for the game.

Definition 4.2. A nonanticipative strategy for Player $I$ on $[t, s](t<s \leq T)$ is a mapping $\alpha$ : $\mathcal{V}_{t, s} \longrightarrow \mathcal{U}_{t, s}$ such that, for any $\mathcal{F}_{r}$-stopping time $S: \Omega \rightarrow[t, s]$ and any $v_{1}, v_{2} \in \mathcal{V}_{t, s}$ with $v_{1} \equiv$ $v_{2}$ on $\llbracket t, S \rrbracket$, it holds $\alpha\left(v_{1}\right) \equiv \alpha\left(v_{2}\right)$ on $\llbracket t, S \rrbracket$. Nonanticipative strategies for Player II on $[t, s]$, $\beta: \mathcal{U}_{t, s} \longrightarrow \mathcal{V}_{t, s}$, are defined similarly. The set of all nonanticipative strategies $\alpha: \mathcal{V}_{t, s} \longrightarrow \mathcal{U}_{t, s}$ for Player I on $[t, s]$ is denoted by $\mathcal{A}_{t, s}$. The set of all nonanticipative strategies $\beta: \mathcal{U}_{t, s} \longrightarrow \mathcal{V}_{t, s}$ for Player II on $[t, s]$ is denoted by $\mathcal{B}_{t, s}$.

(Recall that $\llbracket t, S \rrbracket=\{(r, \omega) \in[0, T] \times \Omega, t \leq r \leq S(\omega)\})$. 
Given the control processes $u(\cdot) \in \mathcal{U}_{t, T}$ and $v(\cdot) \in \mathcal{V}_{t, T}$ we introduce the following associated cost functional

$$
J(t, x ; u, v):=Y_{t}^{t, x ; u, v},(t, x) \in[0, T] \times \mathbb{R}^{n},
$$

where the process $Y^{t, x ; u, v}$ is defined by BSDE (4.5).

Similarly to the proof of Theorem 3.1 we can get that, for any $t \in[0, T]$ and $\zeta \in L^{2}\left(\Omega, \mathcal{F}_{t}, P ; \mathbb{R}^{n}\right)$,

$$
J(t, \zeta ; u, v)=Y_{t}^{t, \zeta ; u, v}, \text { P-a.s.. }
$$

Being particularly interested in the case of a deterministic $\zeta$, i.e., $\zeta=x \in \mathbb{R}^{n}$, we define the lower value function of our stochastic differential game

$$
W(t, x):=\operatorname{essinf}_{\beta \in \mathcal{B}_{t, T}} \operatorname{esssup}_{u \in \mathcal{U}_{t, T}} J(t, x ; u, \beta(u))
$$

and its upper value function

$$
U(t, x):=\operatorname{esssup}_{\alpha \in \mathcal{A}_{t, T}} \operatorname{essinf}_{v \in \mathcal{V}_{t, T}} J(t, x ; \alpha(v), v) .
$$

Remark 4.1. (1) For the convenience of the reader we recall that, given a family of real-valued random variables $\eta_{\alpha}, \alpha \in I$, a random variable $\eta$ is said to be essinf $f_{\alpha \in I} \eta_{\alpha}$, if

i) $\eta \leq \eta_{\alpha}$, P-a.s., for any $\alpha \in I$;

ii) if there is another random variable $\xi$ such that $\xi \leq \eta_{\alpha}$, P-a.s., for any $\alpha \in I$, then $\xi \leq$ $\eta, P$-a.s..

The random variable esssup ${ }_{\alpha \in I} \eta_{\alpha}$ can be introduced now by the relation

$$
\operatorname{essup}_{\alpha \in I} \eta_{\alpha}=-\operatorname{essinf}_{\alpha \in I}\left(-\eta_{\alpha}\right) .
$$

Finally, recall that essinf $f_{\alpha \in I} \eta_{\alpha}=\inf _{n \geq 1} \eta_{\alpha_{n}}$ for some denumerable family $\left(\alpha_{n}\right) \subset I ; \operatorname{esssup}_{\alpha \in I} \eta_{\alpha}$ has the same property.

(2) Obviously, under the assumptions (H4.1)-(H4.2), the lower value function $W(t, x)$ as well as the upper value function $U(t, x)$ are well-defined and a priori they both are bounded $\mathcal{F}_{t}$-measurable random variables. But it turns out that $W(t, x)$ and $U(t, x)$ are even deterministic. Indeed, concentrating on the study of the properties of $W(t, x)$ (the function $U(t, x)$ can be analyzed in a same manner) we can state the following:

Proposition 4.1. For any $[t, x] \in[0, T] \times \mathbb{R}^{n}$, we have $W(t, x)=E[W(t, x)]$, P-a.s.. Identifying $W(t, x)$ with its deterministic version $E[W(t, x)]$ we can consider $W:[0, T] \times \mathbb{R}^{n} \longrightarrow \mathbb{R}$ as a deterministic function.

Remark 4.2. Recall that the fact that the lower and upper value functions defined by Fleming and Souganidis [8] are deterministic is an immediate consequence of their definition. Indeed, for a game over the time interval $[t, T]$ only control processes which are independent of the past $\mathcal{F}_{t}$ are considered as admissible, and since the admissible strategies are supposed to associate admissible control processes of one player with those of the other player, all the associated cost functionals are independent of $\mathcal{F}_{t}$ and hence deterministic.

Proof: Let $H$ denote the Cameron-Martin space of all absolutely continuous elements $h \in \Omega$ whose Radon-Nikodym derivative $\dot{h}$ belongs to $L^{2}\left([0, T], \mathbb{R}^{d}\right)$. 
For any $h \in H$, we define the mapping $\tau_{h} \omega:=\omega+h, \omega \in \Omega$. Obviously, $\tau_{h}: \Omega \rightarrow \Omega$ is a bijection and its law is given by $P \circ\left[\tau_{h}\right]^{-1}=\exp \left\{\int_{0}^{T} \dot{h}_{s} d B_{s}-\frac{1}{2} \int_{0}^{T}\left|\dot{h}_{s}\right|^{2} d s\right\} P$. Let $(t, x) \in[0, T] \times \mathbb{R}^{n}$ be arbitrarily fixed, and put $H_{t}=\{h \in H \mid h(\cdot)=h(\cdot \wedge t)\}$. We split now the proof in the following steps:

$1^{s t}$ step: For any $u \in \mathcal{U}_{t, T}, v \in \mathcal{V}_{t, T}, h \in H_{t}, J(t, x ; u, v)\left(\tau_{h}\right)=J\left(t, x ; u\left(\tau_{h}\right), v\left(\tau_{h}\right)\right)$, P-a.s..

Indeed, we apply the Girsanov transformation to $\operatorname{SDE}(4.1)$ (with $\zeta=x$ ) and compare the obtained equation with the SDE obtained from (4.1) by substituting the transformed control processes $u\left(\tau_{h}\right), v\left(\tau_{h}\right)$ for $u$ and $v$. Then, from the uniqueness of the solution of (4.1) we get $X_{s}^{t, x ; u, v}\left(\tau_{h}\right)=X_{s}^{t, x ; u\left(\tau_{h}\right), v\left(\tau_{h}\right)}$, for any $s \in[t, T]$, P-a.s.. Furthermore, by a similar Girsanov transformation argument we get from the uniqueness of the solution of BSDE (4.5),

$$
\begin{gathered}
Y_{s}^{t, x ; u, v}\left(\tau_{h}\right)=Y_{s}^{t, x ; u\left(\tau_{h}\right), v\left(\tau_{h}\right)}, \text { for any } s \in[t, T], \text { P-a.s., } \\
Z_{s}^{t, x ; u, v}\left(\tau_{h}\right)=Z_{s}^{t, x ; u\left(\tau_{h}\right), v\left(\tau_{h}\right)}, \text { dsdP-a.e. on }[t, T] \times \Omega .
\end{gathered}
$$

That means

$$
J(t, x ; u, v)\left(\tau_{h}\right)=J\left(t, x ; u\left(\tau_{h}\right), v\left(\tau_{h}\right)\right), \text { P-a.s.. }
$$

$2^{\text {nd }}$ step: For $\beta \in \mathcal{B}_{t, T}, h \in H_{t}$, let $\beta^{h}(u):=\beta\left(u\left(\tau_{-h}\right)\right)\left(\tau_{h}\right), u \in \mathcal{U}_{t, T}$. Then $\beta^{h} \in \mathcal{B}_{t, T}$.

Obviously, $\beta^{h}$ maps $\mathcal{U}_{t, T}$ into $\mathcal{V}_{t, T}$. Moreover, this mapping is nonanticipating. Indeed, let $S: \Omega \rightarrow[t, T]$ be an $\mathcal{F}_{r}$-stopping time and $u_{1}, u_{2} \in \mathcal{U}_{t, T}$ with $u_{1} \equiv u_{2}$ on $\llbracket t, S \rrbracket$. Then, obviously, $u_{1}\left(\tau_{-h}\right) \equiv u_{2}\left(\tau_{-h}\right)$ on $\llbracket t, S\left(\tau_{-h}\right) \rrbracket$ (notice that $S\left(\tau_{-h}\right)$ is still a stopping time), and because $\beta \in \mathcal{B}_{t, T}$ we have $\beta\left(u_{1}\left(\tau_{-h}\right)\right) \equiv \beta\left(u_{2}\left(\tau_{-h}\right)\right)$ on $\llbracket t, S\left(\tau_{-h}\right) \rrbracket$. Therefore,

$$
\beta^{h}\left(u_{1}\right)=\beta\left(u_{1}\left(\tau_{-h}\right)\right)\left(\tau_{h}\right) \equiv \beta\left(u_{2}\left(\tau_{-h}\right)\right)\left(\tau_{h}\right)=\beta^{h}\left(u_{2}\right) \text { on } \llbracket t, S \rrbracket .
$$

$3^{\text {rd }}$ step: For all $h \in H_{t}$ and $\beta \in \mathcal{B}_{t, T}$ we have:

$$
\left\{\operatorname{esssup}_{u \in \mathcal{U}_{t, T}} J(t, x ; u, \beta(u))\right\}\left(\tau_{h}\right)=\operatorname{esssup}_{u \in \mathcal{U}_{t, T}}\left\{J(t, x ; u, \beta(u))\left(\tau_{h}\right)\right\} \text {, P-a.s.. }
$$

Indeed, with the notation $I(t, x, \beta):=\operatorname{esssup}_{u \in \mathcal{U}_{t, T}} J(t, x ; u, \beta(u)), \beta \in \mathcal{B}_{t, T}$, we have $I(t, x, \beta) \geq$ $J(t, x ; u, \beta(u))$, and thus $I(t, x, \beta)\left(\tau_{h}\right) \geq J(t, x ; u, \beta(u))\left(\tau_{h}\right)$, P-a.s., for all $u \in \mathcal{U}_{t, T}$. On the other hand, for any random variable $\zeta$ satisfying $\zeta \geq J(t, x ; u, \beta(u))\left(\tau_{h}\right)$, and hence also $\zeta\left(\tau_{-h}\right) \geq$ $J(t, x ; u, \beta(u))$, P-a.s., for all $u \in \mathcal{U}_{t, T}$, we have $\zeta\left(\tau_{-h}\right) \geq I(t, x, \beta)$, P-a.s., i.e., $\zeta \geq I(t, x, \beta)\left(\tau_{h}\right)$, P-a.s.. Consequently,

$$
I(t, x, \beta)\left(\tau_{h}\right)=\operatorname{esssup}_{u \in \mathcal{U}_{t, T}}\left\{J(t, x ; u, \beta(u))\left(\tau_{h}\right)\right\}, \text { P-a.s. }
$$

$4^{\text {th }}$ step: $W(t, x)$ is invariant with respect to the Girsanov transformation $\tau_{h}$, i.e.,

$$
W(t, x)\left(\tau_{h}\right)=W(t, x), \text { P-a.s., for any } h \in H \text {. }
$$

Indeed, similarly to the third step we can show that for all $h \in H_{t}$,

$$
\left\{\operatorname{essinf}_{\beta \in \mathcal{B}_{t, T}} I(t, x ; \beta)\right\}\left(\tau_{h}\right)=\operatorname{essinf}_{\beta \in \mathcal{B}_{t, T}}\left\{I(t, x ; \beta)\left(\tau_{h}\right)\right\}, \text { P-a.s.. }
$$


Then, from the first step to the third step we have, for any $h \in H_{t}$,

$$
\begin{aligned}
W(t, x)\left(\tau_{h}\right) & =\operatorname{essinf}_{\beta \in \mathcal{B}_{t, T}} \operatorname{esssup}_{u \in \mathcal{U}_{t, T}}\left\{J(t, x ; u, \beta(u))\left(\tau_{h}\right)\right\} \\
& =\operatorname{essinf}_{\beta \in \mathcal{B}_{t, T}} \operatorname{esssup}_{u \in \mathcal{U}_{t, T}} J\left(t, x ; u\left(\tau_{h}\right), \beta^{h}\left(u\left(\tau_{h}\right)\right)\right. \\
& =\operatorname{essinf}_{\beta \in \mathcal{B}_{t, T}} \operatorname{esssup}_{u \in \mathcal{U}_{t, T}} J\left(t, x ; u, \beta^{h}(u)\right) \\
& =\operatorname{essinf}_{\beta \in \mathcal{B}_{t, T}} \operatorname{esssup}_{u \in \mathcal{U}_{t, T}} J(t, x ; u, \beta(u)) \\
& =W(t, x), \text { P-a.s. }
\end{aligned}
$$

where we have used $\left\{u\left(\tau_{h}\right) \mid u(\cdot) \in \mathcal{U}_{t, T}\right\}=\mathcal{U}_{t, T},\left\{\beta^{h} \mid \beta \in \mathcal{B}_{t, T}\right\}=\mathcal{B}_{t, T}$ in order to obtain the both latter equalities. Therefore, for any $h \in H_{t}, W(t, x)\left(\tau_{h}\right)=W(t, x)$, P-a.s., and since $W(t, x)$ is $\mathcal{F}_{t}$-measurable, we have this relation even for all $h \in H$.

The result of the $4^{\text {th }}$ step combined with the following auxiliary Lemma 4.1 completes the proof.

Lemma 4.1. Let $\zeta$ be a random variable defined over our classical Wiener space $\left(\Omega, \mathcal{F}_{T}, P\right)$, such that $\zeta\left(\tau_{h}\right)=\zeta$, P-a.s., for any $h \in H$. Then $\zeta=E \zeta, P$-a.s..

Proof: Let $h \in H$ and $A \in \mathcal{B}(\mathbb{R})$. Then,

$$
\begin{aligned}
& E\left[\mathbf{1}_{\{\zeta \in A\}} \exp \left\{\int_{0}^{T} \dot{h}_{s} d B_{s}-\frac{1}{2} \int_{0}^{T}\left|\dot{h}_{s}\right|^{2} d s\right\}\right] \\
= & E\left[\mathbf{1}_{\left\{\zeta\left(\tau_{-h}\right) \in A\right\}} \exp \left\{\int_{0}^{T} \dot{h}_{s} d B_{s}-\frac{1}{2} \int_{0}^{T}\left|\dot{h}_{s}\right|^{2} d s\right\}\right] \\
= & E\left[\mathbf{1}_{\{\zeta \in A\}}\right],
\end{aligned}
$$

from where we deduce that

$$
E\left[\mathbf{1}_{\{\zeta \in A\}} \exp \left\{\int_{0}^{T} \dot{h}_{s} d B_{s}\right\}\right]=E\left[\mathbf{1}_{\{\zeta \in A\}}\right] E\left[\exp \left\{\int_{0}^{T} \dot{h}_{s} d B_{s}\right\}\right],
$$

i.e., for any $\varphi \in L^{2}\left([0, T] ; \mathbb{R}^{d}\right)$,

$$
E\left[\mathbf{1}_{\{\zeta \in A\}} \exp \left\{\int_{0}^{T} \varphi_{s} d B_{s}\right\}\right]=E\left[\mathbf{1}_{\{\zeta \in A\}}\right] E\left[\exp \left\{\int_{0}^{T} \varphi_{s} d B_{s}\right\}\right]
$$

Consequently, taking into consideration the arbitrariness of $A \in \mathcal{B}(\mathbb{R})$ and of $\varphi \in L^{2}\left([0, T] ; \mathbb{R}^{d}\right)$, it follows the independence of $\zeta$ of $B$ and hence of $\mathcal{F}_{T}$, but this is only possible for deterministic $\zeta$.

The first property of the lower value function $W(t, x)$ which we present is an immediate consequence of (4.6) and (4.9).

Lemma 4.2. There exists a constant $C>0$ such that, for all $0 \leq t \leq T, x, x^{\prime} \in \mathbb{R}^{n}$,

$$
\begin{aligned}
& \text { (i) }\left|W(t, x)-W\left(t, x^{\prime}\right)\right| \leq C\left|x-x^{\prime}\right| \\
& \text { (ii) }|W(t, x)| \leq C(1+|x|) .
\end{aligned}
$$

We now discuss (the generalized) dynamic programming principle (DPP) for our stochastic differential game (4.1), (4.5) and (4.9). For this end we have to define the family of (backward) semigroups associated with BSDE (4.5). This notion of stochastic backward semigroups was first introduced by Peng [14] which was applied to study the DPP for stochastic control problems. Our approach adapts Peng's ideas to the framework of stochastic differential games. 
Given the initial data $(t, x)$, a positive number $\delta \leq T-t$, admissible control processes $u(\cdot) \in \mathcal{U}_{t, t+\delta}, v(\cdot) \in \mathcal{V}_{t, t+\delta}$ and a real-valued random variable $\eta \in L^{2}\left(\Omega, \mathcal{F}_{t+\delta}, P ; \mathbb{R}\right)$, we put

$$
G_{s, t+\delta}^{t, x ; u, v}[\eta]:=\tilde{Y}_{s}^{t, x ; u, v}, \quad s \in[t, t+\delta],
$$

where the couple $\left(\tilde{Y}_{s}^{t, x ; u, v}, \tilde{Z}_{s}^{t, x ; u, v}\right)_{t \leq s \leq t+\delta}$ is the solution of the following BSDE with the time horizon $t+\delta$ :

$$
\left\{\begin{aligned}
-d \tilde{Y}_{s}^{t, x ; u, v}= & f\left(s, X_{s}^{t, x ; u, v}, \tilde{Y}_{s}^{t, x ; u, v}, \tilde{Z}_{s}^{t, x ; u, v}, u_{s}, v_{s}\right) d s \\
& -\tilde{Z}_{s}^{t, x ; u, v} d B_{s}, \quad s \in[t, t+\delta], \\
\tilde{Y}_{t+\delta}^{t, x ; u, v}= & \eta,
\end{aligned}\right.
$$

and $X^{t, x ; u, v}$ is the solution of $\operatorname{SDE}(4.1)$. Then, obviously, for the solution $\left(Y^{t, x ; u, v}, Z^{t, x ; u, v}\right)$ of BSDE (4.5) we have

$$
G_{t, T}^{t, x ; u, v}\left[\Phi\left(X_{T}^{t, x ; u, v}\right)\right]=G_{t, t+\delta}^{t, x ; u, v}\left[Y_{t+\delta}^{t, x ; u, v}\right]
$$

Moreover,

$$
\begin{aligned}
J(t, x ; u, v) & =Y_{t}^{t, x ; u, v}=G_{t, T}^{t, x ; u, v}\left[\Phi\left(X_{T}^{t, x ; u, v}\right)\right]=G_{t, t+\delta}^{t, x ; u, v}\left[Y_{t+\delta}^{t, x ; u, v}\right] \\
& =G_{t, t+\delta}^{t, x ; u, v}\left[J\left(t+\delta, X_{t+\delta}^{t, x ; u, v} ; u, v\right)\right] .
\end{aligned}
$$

Remark 4.3. When $f$ is independent of $(y, z)$ it holds that

$$
G_{s, t+\delta}^{t, x ; u, v}[\eta]=E\left[\eta+\int_{s}^{t+\delta} f\left(r, X_{r}^{t, x ; u, v}, u_{r}, v_{r}\right) d r \mid \mathcal{F}_{s}\right], \quad s \in[t, t+\delta] .
$$

Theorem 4.1. Under the assumptions (H4.1) and (H4.2), the lower value function $W(t, x)$ obeys the following DPP : For any $0 \leq t<t+\delta \leq T, x \in \mathbb{R}^{n}$,

$$
W(t, x)=\operatorname{essinf}_{\beta \in \mathcal{B}_{t, t+\delta}} \operatorname{esssup}_{u \in \mathcal{U}_{t, t+\delta}} G_{t, t+\delta}^{t, x ; u, \beta(u)}\left[W\left(t+\delta, X_{t+\delta}^{t, x ; u, \beta(u)}\right)\right] .
$$

Proof: To simplify notations we put

$$
W_{\delta}(t, x)=\operatorname{essinf}_{\beta \in \mathcal{B}_{t, t+\delta}} \operatorname{esssup}_{u \in \mathcal{U}_{t, t+\delta}} G_{t, t+\delta}^{t, x ; u, \beta(u)}\left[W\left(t+\delta, X_{t+\delta}^{t, x ; u, \beta(u)}\right)\right] .
$$

The proof that $W_{\delta}(t, x)$ coincides with $W(t, x)$ will be split into a sequel of lemmata which all are supposed to satisfy (H4.1) and (H4.2).

Lemma 4.3. $W_{\delta}(t, x)$ is deterministic.

The proof of this lemma uses the same ideas as that of Proposition 4.1 so that it can be omitted here.

Lemma 4.4. $W_{\delta}(t, x) \leq W(t, x)$.

Proof: Let $\beta \in \mathcal{B}_{t, T}$ be arbitrarily fixed. Then, given a $u_{2}(\cdot) \in \mathcal{U}_{t+\delta, T}$, we define as follows the restriction $\beta_{1}$ of $\beta$ to $\mathcal{U}_{t+\delta, T}$ :

$$
\beta_{1}\left(u_{1}\right):=\left.\beta\left(u_{1} \oplus u_{2}\right)\right|_{[t, t+\delta]}, \quad u_{1}(\cdot) \in \mathcal{U}_{t, t+\delta},
$$

where $u_{1} \oplus u_{2}:=u_{1} \mathbf{1}_{[t, t+\delta]}+u_{2} \mathbf{1}_{(t+\delta, T]}$ extends $u_{1}(\cdot)$ to an element of $\mathcal{U}_{t, T}$. It is easy to check that $\beta_{1} \in \mathcal{B}_{t, t+\delta}$. Moreover, from the nonanticipativity property of $\beta$ we deduce that $\beta_{1}$ is independent of the special choice of $u_{2}(\cdot) \in \mathcal{U}_{t+\delta, T}$. Consequently, from the definition of $W_{\delta}(t, x)$,

$$
W_{\delta}(t, x) \leq \operatorname{esssup}_{u_{1} \in \mathcal{U}_{t, t+\delta}} G_{t, t+\delta}^{t, x ; u_{1}, \beta_{1}\left(u_{1}\right)}\left[W\left(t+\delta, X_{t+\delta}^{t, x ; u_{1}, \beta_{1}\left(u_{1}\right)}\right)\right], \text { P-a.s.. }
$$


We use the notation $I_{\delta}(t, x, u, v):=G_{t, t+\delta}^{t, x ; u, v}\left[W\left(t+\delta, X_{t+\delta}^{t, x ; u, v}\right)\right]$ and notice that there exists a sequence $\left\{u_{i}^{1}, i \geq 1\right\} \subset \mathcal{U}_{t, t+\delta}$ such that

$$
I_{\delta}\left(t, x, \beta_{1}\right):=\operatorname{esssup}_{u_{1} \in \mathcal{U}_{t, t+\delta}} I_{\delta}\left(t, x, u_{1}, \beta_{1}\left(u_{1}\right)\right)=\sup _{i \geq 1} I_{\delta}\left(t, x, u_{i}^{1}, \beta_{1}\left(u_{i}^{1}\right)\right), \quad \text { P-a.s.. }
$$

For any $\varepsilon>0$, we put $\widetilde{\Gamma}_{i}:=\left\{I_{\delta}\left(t, x, \beta_{1}\right) \leq I_{\delta}\left(t, x, u_{i}^{1}, \beta_{1}\left(u_{i}^{1}\right)\right)+\varepsilon\right\} \in \mathcal{F}_{t}, i \geq 1$. Then $\Gamma_{1}:=$ $\widetilde{\Gamma}_{1}, \Gamma_{i}:=\widetilde{\Gamma}_{i} \backslash\left(\cup_{l=1}^{i-1} \widetilde{\Gamma}_{l}\right) \in \mathcal{F}_{t}, i \geq 2$, form an $\left(\Omega, \mathcal{F}_{t}\right)$-partition, and $u_{1}^{\varepsilon}:=\sum_{i>1} \mathbf{1}_{\Gamma_{i}} u_{i}^{1}$ belongs obviously to $\mathcal{U}_{t, t+\delta}$. Moreover, from the nonanticipativity of $\beta_{1}$ we have $\beta_{1}\left(u_{1}^{\varepsilon}\right)=\sum_{i \geq 1} \mathbf{1}_{\Gamma_{i}} \beta_{1}\left(u_{i}^{1}\right)$, and from the uniqueness of the solution of the FBSDE, we deduce that $I_{\delta}\left(t, x, u_{1}^{\varepsilon}, \beta_{1}\left(u_{1}^{\varepsilon}\right)\right)=$ $\sum_{i \geq 1} \mathbf{1}_{\Gamma_{i}} I_{\delta}\left(t, x, u_{i}^{1}, \beta_{1}\left(u_{i}^{1}\right)\right)$, P-a.s.. Hence,

$$
\begin{aligned}
W_{\delta}(t, x) \leq I_{\delta}\left(t, x, \beta_{1}\right) & \leq \sum_{i \geq 1} \mathbf{1}_{\Gamma_{i}} I_{\delta}\left(t, x, u_{i}^{1}, \beta_{1}\left(u_{i}^{1}\right)\right)+\varepsilon=I_{\delta}\left(t, x, u_{1}^{\varepsilon}, \beta_{1}\left(u_{1}^{\varepsilon}\right)\right)+\varepsilon \\
& =G_{t, t+\delta}^{t, x ; u_{1}^{\varepsilon}, \beta_{1}\left(u_{1}^{\varepsilon}\right)}\left[W\left(t+\delta, X_{t+\delta}^{t, x ; u_{1}^{\varepsilon}, \beta_{1}\left(u_{1}^{\varepsilon}\right)}\right)\right]+\varepsilon, \text { P-a.s.. }
\end{aligned}
$$

On the other hand, using the fact that $\beta_{1}(\cdot):=\beta\left(\cdot \oplus u_{2}\right) \in \mathcal{B}_{t, t+\delta}$ does not depend on $u_{2}(\cdot) \in \mathcal{U}_{t+\delta, T}$ we can define $\beta_{2}\left(u_{2}\right):=\left.\beta\left(u_{1}^{\varepsilon} \oplus u_{2}\right)\right|_{[t+\delta, T]}$, for all $u_{2}(\cdot) \in \mathcal{U}_{t+\delta, T}$. The such defined $\beta_{2}: \mathcal{U}_{t+\delta, T} \rightarrow$ $\mathcal{V}_{t+\delta, T}$ belongs to $\mathcal{B}_{t+\delta, T}$ since $\beta \in \mathcal{B}_{t, T}$. Therefore, from the definition of $W(t+\delta, y)$ we have, for any $y \in \mathbb{R}^{n}$,

$$
W(t+\delta, y) \leq \operatorname{esssup}_{u_{2} \in \mathcal{U}_{t+\delta, T}} J\left(t+\delta, y ; u_{2}, \beta_{2}\left(u_{2}\right)\right), \text { P-a.s.. }
$$

Finally, because there exists a constant $C \in \mathbb{R}$ such that

(i) $\left|W(t+\delta, y)-W\left(t+\delta, y^{\prime}\right)\right| \leq C\left|y-y^{\prime}\right|$, for any $y, y^{\prime} \in \mathbb{R}^{n}$;

(ii) $\left|J\left(t+\delta, y, u_{2}, \beta_{2}\left(u_{2}\right)\right)-J\left(t+\delta, y^{\prime}, u_{2}, \beta_{2}\left(u_{2}\right)\right)\right| \leq C\left|y-y^{\prime}\right|$, P-a.s.,

for any $u_{2} \in \mathcal{U}_{t+\delta, T}$,

(see Lemma 4.2-(i) and (4.6)-(i)) we can show by approximating $X_{t+\delta}^{t, x ; u_{1}^{\varepsilon}, \beta_{1}\left(u_{1}^{\varepsilon}\right)}$ that

$$
W\left(t+\delta, X_{t+\delta}^{t, x ; u_{1}^{\varepsilon}, \beta_{1}\left(u_{1}^{\varepsilon}\right)}\right) \leq \operatorname{esssup}_{u_{2} \in \mathcal{U}_{t+\delta, T}} J\left(t+\delta, X_{t+\delta}^{t, x ; u_{1}^{\varepsilon}, \beta_{1}\left(u_{1}^{\varepsilon}\right)} ; u_{2}, \beta_{2}\left(u_{2}\right)\right), \text { P-a.s.. }
$$

To estimate the right side of the latter inequality we note that there exists some sequence $\left\{u_{j}^{2}, j \geq\right.$ 1\} $\subset \mathcal{U}_{t+\delta, T}$ such that

$$
\operatorname{esssup}_{u_{2} \in \mathcal{U}_{t+\delta, T}} J\left(t+\delta, X_{t+\delta}^{t, x ; u_{1}^{\varepsilon}, \beta_{1}\left(u_{1}^{\varepsilon}\right)} ; u_{2}, \beta_{2}\left(u_{2}\right)\right)=\sup _{j \geq 1} J\left(t+\delta, X_{t+\delta}^{t, x ; u_{1}^{\varepsilon}, \beta_{1}\left(u_{1}^{\varepsilon}\right)} ; u_{j}^{2}, \beta_{2}\left(u_{j}^{2}\right)\right), \text { P-a.s.. }
$$

Then, putting

$\widetilde{\Delta}_{j}:=\left\{\operatorname{esssup}_{u_{2} \in \mathcal{U}_{t+\delta, T}} J\left(t+\delta, X_{t+\delta}^{t, x ; u_{1}^{\varepsilon}, \beta_{1}\left(u_{1}^{\varepsilon}\right)} ; u_{2}, \beta_{2}\left(u_{2}\right)\right) \leq J\left(t+\delta, X_{t+\delta}^{t, x ; u_{1}^{\varepsilon}, \beta_{1}\left(u_{1}^{\varepsilon}\right)} ; u_{j}^{2}, \beta_{2}\left(u_{j}^{2}\right)\right)+\varepsilon\right\} \in$ $\mathcal{F}_{t+\delta}, j \geq 1$; we have with $\Delta_{1}:=\widetilde{\Delta}_{1}, \Delta_{j}:=\widetilde{\Delta}_{j} \backslash\left(\cup_{l=1}^{j-1} \widetilde{\Delta}_{l}\right) \in \mathcal{F}_{t+\delta}, j \geq 2$, an $\left(\Omega, \mathcal{F}_{t+\delta}\right)$-partition and $u_{2}^{\varepsilon}:=\sum_{j \geq 1} \mathbf{1}_{\Delta_{j}} u_{j}^{2} \in \mathcal{U}_{t+\delta, T}$. From the nonanticipativity of $\beta_{2}$ we have $\beta_{2}\left(u_{2}^{\varepsilon}\right)=\sum_{j \geq 1} \mathbf{1}_{\Delta_{j}} \beta_{2}\left(u_{j}^{2}\right)$ and from the definition of $\beta_{1}, \beta_{2}$ we know that $\beta\left(u_{1}^{\varepsilon} \oplus u_{2}^{\varepsilon}\right)=\beta_{1}\left(u_{1}^{\varepsilon}\right) \oplus \beta_{2}\left(u_{2}^{\varepsilon}\right)$. Thus, again from the uniqueness of the solution of our FBSDE, we get

$$
\begin{aligned}
& J\left(t+\delta, X_{t+\delta}^{t, x ; u_{1}^{\varepsilon}, \beta_{1}\left(u_{1}^{\varepsilon}\right)} ; u_{2}^{\varepsilon}, \beta_{2}\left(u_{2}^{\varepsilon}\right)\right)=Y_{t+\delta}^{t+\delta, X_{t+\delta}^{t, x ; u_{1}^{\varepsilon}, \beta_{1}\left(u_{1}^{\varepsilon}\right)} ; u_{2}^{\varepsilon}, \beta_{2}\left(u_{2}^{\varepsilon}\right)} \\
& =\sum_{j \geq 1} \mathbf{1}_{\Delta_{j}} Y_{t+\delta}^{t+\delta, X_{t+\delta}^{t, x ; u_{1}^{\varepsilon}, \beta_{1}\left(u_{1}^{\varepsilon}\right)} ; u_{j}^{2}, \beta_{2}\left(u_{j}^{2}\right)} \\
& =\sum_{j \geq 1} \mathbf{1}_{\Delta_{j}} J\left(t+\delta, X_{t+\delta}^{t, x ; u_{1}^{\varepsilon}, \beta_{1}\left(u_{1}^{\varepsilon}\right)} ; u_{j}^{2}, \beta_{2}\left(u_{j}^{2}\right)\right) \text {, P-a.s.. }
\end{aligned}
$$


Consequently,

$$
\begin{aligned}
& W\left(t+\delta, X_{t+\delta}^{t, x ; u_{1}^{\varepsilon}, \beta_{1}\left(u_{1}^{\varepsilon}\right)}\right) \leq \operatorname{esssup}_{u_{2} \in \mathcal{U}_{t+\delta, T}} J\left(t+\delta, X_{t+\delta}^{t, x ; u_{1}^{\varepsilon}, \beta_{1}\left(u_{1}^{\varepsilon}\right)} ; u_{2}, \beta_{2}\left(u_{2}\right)\right) \\
& \leq \sum_{j \geq 1} \mathbf{1}_{\Delta_{j}} Y_{t+\delta}^{t, x ; u_{1}^{\varepsilon} \oplus u_{j}^{2}, \beta\left(u_{1}^{\varepsilon} \oplus u_{j}^{2}\right)}+\varepsilon \\
& =Y_{t+\delta}^{t, x ; u_{1}^{\varepsilon} \oplus u_{2}^{\varepsilon}, \beta\left(u_{1}^{\varepsilon} \oplus u_{2}^{\varepsilon}\right)}+\varepsilon \\
& =Y_{t+\delta}^{t, x ; u^{\varepsilon}, \beta\left(u^{\varepsilon}\right)}+\varepsilon, \text { P-a.s., }
\end{aligned}
$$

where $u^{\varepsilon}:=u_{1}^{\varepsilon} \oplus u_{2}^{\varepsilon} \in \mathcal{U}_{t, T}$. From (4.17), (4.19), Lemma 2.2 (comparison theorem for BSDEs) and Lemma 2.3 we have

$$
\begin{aligned}
W_{\delta}(t, x) & \leq G_{t, t+u_{1}^{\varepsilon}, \beta_{1}\left(u_{1}^{\varepsilon}\right)}^{t, \delta}\left[Y_{t+\delta}^{t, x ; u^{\varepsilon}, \beta\left(u^{\varepsilon}\right)}+\varepsilon\right]+\varepsilon \\
& \leq G_{t, t+u_{1}^{\varepsilon}, \beta_{1}\left(u_{1}^{\varepsilon}\right)}^{t, \delta}\left[Y_{t+\delta ; u^{\varepsilon}, \beta\left(u^{\varepsilon}\right)}^{t, \delta}\right]+(C+1) \varepsilon \\
& =G_{t, t+u^{\varepsilon}, \beta\left(u^{\varepsilon}\right)}^{t, \delta}\left[Y_{t+\delta}^{t, x}, \beta u^{\varepsilon}, \beta\left(u^{\varepsilon}\right)\right]+(C+1) \varepsilon \\
& =Y_{t}^{t, x ; u^{\varepsilon}, \beta\left(u^{\varepsilon}\right)}+(C+1) \varepsilon \\
& \leq \operatorname{esssup}_{u \in \mathcal{U}_{t, T}} Y_{t}^{t, x ; u, \beta(u)}+(C+1) \varepsilon, \text { P-a.s.. }
\end{aligned}
$$

Since $\beta \in \mathcal{B}_{t, T}$ has been arbitrarily chosen we have (4.20) for all $\beta \in \mathcal{B}_{t, T}$. Therefore,

$$
W_{\delta}(t, x) \leq \operatorname{essinf}_{\beta \in \mathcal{B}_{t, T}} \operatorname{esssup}_{u \in \mathcal{U}_{t, T}} Y_{t}^{t, x ; u, \beta(u)}+(C+1) \varepsilon=W(t, x)+(C+1) \varepsilon .
$$

Finally, letting $\varepsilon \downarrow 0$, we get $W_{\delta}(t, x) \leq W(t, x)$.

Lemma 4.5. $W(t, x) \leq W_{\delta}(t, x)$.

Proof: We continue to use the notations introduced above, from the definition of $W_{\delta}(t, x)$ we have

$$
\begin{aligned}
W_{\delta}(t, x) & =\operatorname{essinf}_{\beta_{1} \in \mathcal{B}_{t, t+\delta}} \operatorname{esssup}_{u_{1} \in \mathcal{U}_{t, t+\delta}} G_{t, t+\delta}^{t, x ; u_{1}, \beta_{1}\left(u_{1}\right)}\left[W\left(t+\delta, X_{t+\delta}^{t, x ; u_{1}, \beta_{1}\left(u_{1}\right)}\right)\right] \\
& =\operatorname{essinf}_{\beta_{1} \in \mathcal{B}_{t, t+\delta}} I_{\delta}\left(t, x, \beta_{1}\right)
\end{aligned}
$$

and, for some sequence $\left\{\beta_{i}^{1}, i \geq 1\right\} \subset \mathcal{B}_{t, t+\delta}$,

$$
W_{\delta}(t, x)=\inf _{i \geq 1} I_{\delta}\left(t, x, \beta_{i}^{1}\right), \text { P-a.s.. }
$$

For any $\varepsilon>0$, we let $\widetilde{\Lambda}_{i}:=\left\{I_{\delta}\left(t, x, \beta_{i}^{1}\right)-\varepsilon \leq W_{\delta}(t, x)\right\} \in \mathcal{F}_{t}, i \geq 1, \Lambda_{1}:=\widetilde{\Lambda}_{1}$ and $\Lambda_{i}:=$ $\widetilde{\Lambda}_{i} \backslash\left(\cup_{l=1}^{i-1} \widetilde{\Lambda}_{l}\right) \in \mathcal{F}_{t}, i \geq 2$. Then $\left\{\Lambda_{i}, i \geq 1\right\}$ is an $\left(\Omega, \mathcal{F}_{t}\right)$-partition, $\beta_{1}^{\varepsilon}:=\sum_{i \geq 1} \mathbf{1}_{\Lambda_{i}} \beta_{i}^{1}$ belongs to $\mathcal{B}_{t, t+\delta}$, and from the uniqueness of the solution of our FBSDE we conclude that $I_{\delta}\left(t, x, u_{1}, \beta_{1}^{\varepsilon}\left(u_{1}\right)\right)=$ $\sum_{i \geq 1} \mathbf{1}_{\Lambda_{i}} I_{\delta}\left(t, x, u_{1}, \beta_{i}^{1}\left(u_{1}\right)\right)$, P-a.s., for all $u_{1}(\cdot) \in \mathcal{U}_{t, t+\delta}$. Hence,

$$
\begin{aligned}
W_{\delta}(t, x) & \geq \sum_{i \geq 1} \mathbf{1}_{\Lambda_{i}} I_{\delta}\left(t, x, \beta_{i}^{1}\right)-\varepsilon \\
& \geq \sum_{i \geq 1} \mathbf{1}_{\Lambda_{i}} I_{\delta}\left(t, x, u_{1}, \beta_{i}^{1}\left(u_{1}\right)\right)-\varepsilon \\
& =I_{\delta}\left(t, x, u_{1}, \beta_{1}^{\varepsilon}\left(u_{1}\right)\right)-\varepsilon \\
& =G_{t, t+\delta}^{t, x ; u_{1}, \beta_{1}^{\varepsilon}\left(u_{1}\right)}\left[W\left(t+\delta, X_{t+\delta}^{t, x ; u_{1}, \beta_{1}^{\varepsilon}\left(u_{1}\right)}\right)\right]-\varepsilon, \text { P-a.s., for all } u_{1} \in \mathcal{U}_{t, t+\delta} .
\end{aligned}
$$

On the other hand, from the definition of $W(t+\delta, y)$, with the same technique as before, we deduce that, for any $y \in \mathbb{R}^{n}$, there exists $\beta_{y}^{\varepsilon} \in \mathcal{B}_{t+\delta, T}$ such that

$$
W(t+\delta, y) \geq \operatorname{esssup}_{u_{2} \in \mathcal{U}_{t+\delta, T}} J\left(t+\delta, y ; u_{2}, \beta_{y}^{\varepsilon}\left(u_{2}\right)\right)-\varepsilon, \text { P-a.s.. }
$$


Let $\left\{O_{i}\right\}_{i \geq 1} \subset \mathcal{B}\left(\mathbb{R}^{n}\right)$ be a decomposition of $\mathbb{R}^{n}$ such that $\sum_{i \geq 1} O_{i}=\mathbb{R}^{n}$ and $\operatorname{diam}\left(O_{i}\right) \leq \varepsilon, i \geq 1$. And let $y_{i}$ be an arbitrarily fixed element of $O_{i}, i \geq 1$. Defining $\left[X_{t+\delta}^{t, x ; u_{1}, \beta_{1}^{\varepsilon}\left(u_{1}\right)}\right]:=\sum_{i \geq 1} y_{i} \mathbf{1}_{\left\{X_{t+\delta}^{t, x ; u_{1}, \beta_{1}^{\varepsilon}\left(u_{1}\right)} \in O_{i}\right\}}$, we have

$$
\left|X_{t+\delta}^{t, x ; u_{1}, \beta_{1}^{\varepsilon}\left(u_{1}\right)}-\left[X_{t+\delta}^{t, x ; u_{1}, \beta_{1}^{\varepsilon}\left(u_{1}\right)}\right]\right| \leq \varepsilon, \text { everywhere on } \Omega \text {, for all } u_{1} \in \mathcal{U}_{t, t+\delta} .
$$

Moreover, for each $y_{i}$, there exists some $\beta_{y_{i}}^{\varepsilon} \in \mathcal{B}_{t+\delta, T}$ such that (4.23) holds, and, clearly, $\beta_{u_{1}}^{\varepsilon}:=$ $\sum_{i \geq 1} \mathbf{1}_{\left\{X_{t+\delta}^{t, x ; u_{1}, \beta_{1}^{\varepsilon}\left(u_{1}\right)} \in O_{i}\right\}} \beta_{y_{i}}^{\varepsilon} \in \mathcal{B}_{t+\delta, T}$.

Now we can define the new strategy $\beta^{\varepsilon}(u):=\beta_{1}^{\varepsilon}\left(u_{1}\right) \oplus \beta_{u_{1}}^{\varepsilon}\left(u_{2}\right), u \in \mathcal{U}_{t, T}$, where $u_{1}=$ $\left.u\right|_{[t, t+\delta]}, u_{2}=\left.u\right|_{(t+\delta, T]}$ (restriction of $u$ to $[t, t+\delta] \times \Omega$ and $(t+\delta, T] \times \Omega$, resp.). Obviously, $\beta^{\varepsilon}$ maps $\mathcal{U}_{t, T}$ into $\mathcal{V}_{t, T}$. Moreover, $\beta^{\varepsilon}$ is nonanticipating: Indeed, let $S: \Omega \longrightarrow[t, T]$ be an $\mathcal{F}_{r}$-stopping time and $u, u^{\prime} \in \mathcal{U}_{t, T}$ be such that $u \equiv u^{\prime}$ on $\llbracket t, S \rrbracket$. Decomposing $u, u^{\prime}$ into $u_{1}, u_{1}^{\prime} \in \mathcal{U}_{t, t+\delta}, u_{2}, u_{2}^{\prime} \in$ $\mathcal{U}_{t+\delta, T}$ such that $u=u_{1} \oplus u_{2}$ and $u^{\prime}=u_{1}^{\prime} \oplus u_{2}^{\prime}$. We have $u_{1} \equiv u_{1}^{\prime}$ on $\llbracket t, S \wedge(t+\delta) \rrbracket$ from where we get $\beta_{1}^{\varepsilon}\left(u_{1}\right) \equiv \beta_{1}^{\varepsilon}\left(u_{1}^{\prime}\right)$ on $\llbracket t, S \wedge(t+\delta) \rrbracket$ (recall that $\beta_{1}^{\varepsilon}$ is nonanticipating). On the other hand, $u_{2} \equiv u_{2}^{\prime}$ on $\rrbracket t+\delta, S \vee(t+\delta) \rrbracket(\subset(t+\delta, T] \times\{S>t+\delta\})$, and on $\{S>t+\delta\}$ we have $X_{t+\delta}^{t, x ; u_{1}, \beta_{1}^{\varepsilon}\left(u_{1}\right)}=$ $X_{t+\delta}^{t, x ; u_{1}^{\prime}, \beta_{1}^{\varepsilon}\left(u_{1}^{\prime}\right)}$. Consequently, from our definition, $\beta_{u_{1}}^{\varepsilon}=\beta_{u_{1}^{\prime}}^{\varepsilon}$ on $\{S>t+\delta\}$ and $\beta_{u_{1}}^{\varepsilon}\left(u_{2}\right) \equiv \beta_{u_{1}^{\prime}}^{\varepsilon}\left(u_{2}^{\prime}\right)$ on $\rrbracket t+\delta, S \vee(t+\delta) \rrbracket$. This yields $\beta^{\varepsilon}(u)=\beta_{1}^{\varepsilon}\left(u_{1}\right) \oplus \beta_{u_{1}}^{\varepsilon}\left(u_{2}\right) \equiv \beta_{1}^{\varepsilon}\left(u_{1}^{\prime}\right) \oplus \beta_{u_{1}^{\prime}}^{\varepsilon}\left(u_{2}^{\prime}\right)=\beta^{\varepsilon}\left(u^{\prime}\right)$ on $\llbracket t, S \rrbracket$, from where it follows that $\beta^{\varepsilon} \in \mathcal{B}_{t, T}$.

Let now $u \in \mathcal{U}_{t, T}$ be arbitrarily chosen and decomposed into $u_{1}=\left.u\right|_{[t, t+\delta]} \in \mathcal{U}_{t, t+\delta}$ and $u_{2}=\left.u\right|_{(t+\delta, T]} \in \mathcal{U}_{t+\delta, T}$. Then, from (4.22), (4.18)-(i), (4.24) and the lemmata 2.2 (comparison theorem) and 2.3 we obtain,

$$
\begin{aligned}
W_{\delta}(t, x) & \geq G_{t, t+\delta}^{t, x ; u_{1}, \beta_{1}^{\varepsilon}\left(u_{1}\right)}\left[W\left(t+\delta, X_{t+\delta}^{t, x ; u_{1}, \beta_{1}^{\varepsilon}\left(u_{1}\right)}\right)\right]-\varepsilon \\
& \geq G_{t, t+u_{1}, \beta_{1}^{\varepsilon}\left(u_{1}\right)}^{t, x}\left[W\left(t+\delta,\left[X_{t+\delta}^{t, x ; u_{1}, \beta_{1}^{\varepsilon}\left(u_{1}\right)}\right]\right)-C \varepsilon\right]-\varepsilon \\
& \geq G_{t, t+t+\delta}^{t, x ; \beta_{1}\left(u_{1}\right)}\left[W\left(t+\delta,\left[X_{t+\delta}^{t, x ; u_{1}, \beta_{1}^{\varepsilon}\left(u_{1}\right)}\right]\right)\right]-C \varepsilon \\
& =G_{t, t+\delta}^{t, x ; u_{1}, \beta_{1}^{\varepsilon}\left(u_{1}\right)}\left[\sum_{i \geq 1} \mathbf{1}_{\left\{X_{t+\delta}^{t, x ; u_{1}, \beta_{1}^{\varepsilon}\left(u_{1}\right)} \in O_{i}\right\}} W\left(t+\delta, y_{i}\right)\right]-C \varepsilon, \quad \text { P-a.s.. }
\end{aligned}
$$

Furthermore, from (4.23), (4.18)-(ii), (4.24), Lemmata 2.2 (comparison theorem) and 2.3, we have,

$$
\begin{aligned}
W_{\delta}(t, x) & \geq G_{t, t+\delta}^{t, x ; u_{1}, \beta_{1}^{\varepsilon}\left(u_{1}\right)}\left[\sum_{i \geq 1} \mathbf{1}_{\left\{X_{t+\delta}^{t, x ; u_{1}, \beta_{1}^{\varepsilon}\left(u_{1}\right)} \in O_{i}\right\}} J\left(t+\delta, y_{i} ; u_{2}, \beta_{y_{i}}^{\varepsilon}\left(u_{2}\right)\right)-\varepsilon\right]-C \varepsilon \\
& \geq G_{t, t+\delta}^{t, x ; u_{1}, \beta_{1}^{\varepsilon}\left(u_{1}\right)}\left[\sum_{i \geq 1} \mathbf{1}_{\left\{X_{t+\delta}^{t, x ; u_{1}, \beta_{1}^{\varepsilon}\left(u_{1}\right)} \in O_{i}\right\}} J\left(t+\delta, y_{i} ; u_{2}, \beta_{y_{i}}^{\varepsilon}\left(u_{2}\right)\right)\right]-C \varepsilon \\
& =G_{t, t+\delta}^{t, x ; u_{1}, \beta_{1}^{\varepsilon}\left(u_{1}\right)}\left[J\left(t+\delta,\left[X_{t+\delta}^{t, x ; u_{1}, \beta_{1}^{\varepsilon}\left(u_{1}\right)}\right] ; u_{2}, \beta_{u_{1}}^{\varepsilon}\left(u_{2}\right)\right)\right]-C \varepsilon \\
& \geq G_{t, t+u_{1}, \beta_{1}^{\varepsilon}\left(u_{1}\right)}^{t, x}\left[J\left(t+\delta, X_{t+\delta}^{t, x ; u_{1}, \beta_{1}^{\varepsilon}\left(u_{1}\right)} ; u_{2}, \beta_{u_{1}}^{\varepsilon}\left(u_{2}\right)\right)-C \varepsilon\right]-C \varepsilon \\
& \geq G_{t, t+\delta}^{t, x ; \beta_{1}, \beta_{1}^{\varepsilon}\left(u_{1}\right)}\left[J\left(t+\delta, X_{t+\delta}^{t, x ; u_{1}, \beta_{1}^{\varepsilon}\left(u_{1}\right)} ; u_{2}, \beta_{u_{1}}^{\varepsilon}\left(u_{2}\right)\right)\right]-C \varepsilon \\
& =G_{t, t+\delta}^{t, x ; \beta^{\varepsilon}(u)}\left[Y_{t+\delta}^{t, x, u, \beta^{\varepsilon}(u)}\right]-C \varepsilon \\
& =Y_{t}^{t, x ; u, \beta^{\varepsilon}(u)}-C \varepsilon, \mathrm{P}-\text { a.s. }, \text { for any } u \in \mathcal{U}_{t, T} .
\end{aligned}
$$

Consequently,

$$
\begin{aligned}
W_{\delta}(t, x) & \geq \operatorname{esssup}_{u \in \mathcal{U}_{t, T}} J\left(t, x ; u, \beta^{\varepsilon}(u)\right)-C \varepsilon \\
& \geq \operatorname{essinf}_{\beta \in \mathcal{B}_{t, T}} \operatorname{esssup}_{u \in \mathcal{U}_{t, T}} J(t, x ; u, \beta(u))-C \varepsilon \\
& =W(t, x)-C \varepsilon, \text { P-a.s.. }
\end{aligned}
$$

Finally, letting $\varepsilon \downarrow 0$ we get $W_{\delta}(t, x) \geq W(t, x)$. The proof is complete. 
Remark 4.4. (i) From the inequalities (4.17) and (4.22) we see that for all $(t, x) \in[0, T] \times \mathbb{R}^{n}$, $\delta>0$ with $0<\delta \leq T-t$ and $\varepsilon>0$, it holds:

a) For every $\beta \in \mathcal{B}_{t, t+\delta}$, there exists some $u^{\varepsilon}(\cdot) \in \mathcal{U}_{t, t+\delta}$ such that

$$
W(t, x)\left(=W_{\delta}(t, x)\right) \leq G_{t, t+\delta}^{t, x ; u^{\varepsilon}, \beta\left(u^{\varepsilon}\right)}\left[W\left(t+\delta, X_{t+\delta}^{t, x ; u^{\varepsilon}, \beta\left(u^{\varepsilon}\right)}\right)\right]+\varepsilon, \quad P-a . s . .
$$

b) There exists some $\beta^{\varepsilon} \in \mathcal{B}_{t, t+\delta}$ such that, for all $u \in \mathcal{U}_{t, t+\delta}$,

$$
W(t, x)\left(=W_{\delta}(t, x)\right) \geq G_{t, t+\delta}^{t, x ; u, \beta^{\varepsilon}(u)}\left[W\left(t+\delta, X_{t+\delta}^{t, x ; u, \beta^{\varepsilon}(u)}\right)\right]-\varepsilon, \quad P-a . s . .
$$

(ii) Recall that the lower value function $W$ is deterministic. Thus, by taking the expectation on both sides of (4.28) and (4.29) we can show that

$$
W(t, x)=\inf _{\beta \in \mathcal{B}_{t, T}} \sup _{u \in \mathcal{U}_{t, T}} E[J(t, x ; u, \beta(u))] .
$$

In analogy we also have

$$
U(t, x)=\sup _{\alpha \in \mathcal{A}_{t, T}} \inf _{v \in \mathcal{V}_{t, T}} E[J(t, x ; \alpha(v), v)] .
$$

The above formulas look similar to the definitions of the lower and the upper value functions defined by Fleming and Souganidis [8] for the case of $f$ being independent of $(y, z)$. However, they consider only control processes which are independent of the past $\mathcal{F}_{t}$. In Remark 6.3 we will come back to this comparison and identify their value functions with ours for such coefficient $f$.

In Lemma 4.2 we have already seen that the lower value function $W(t, x)$ is Lipschitz continuous in $x$, uniformly in $t$. With the help of Theorem 4.1 we can now also study the continuity properties of $W(t, x)$ in $t$.

Theorem 4.2. Let us suppose that the assumptions (H4.1) and (H4.2) hold. Then the lower value function $W(t, x)$ is $\frac{1}{2}-H$ ölder continuous in $t$ : There exists a constant $C$ such that, for every $x \in \mathbb{R}^{n}, t, t^{\prime} \in[0, T]$,

$$
\left|W(t, x)-W\left(t^{\prime}, x\right)\right| \leq C(1+|x|)\left|t-t^{\prime}\right|^{\frac{1}{2}}
$$

Proof: Let $(t, x) \in[0, T] \times \mathbb{R}^{n}$ and $\delta>0$ be arbitrarily given such that $0<\delta \leq T-t$. Our objective is to prove the following inequality by using (4.28) and (4.29):

$$
-C(1+|x|) \delta^{\frac{1}{2}} \leq W(t, x)-W(t+\delta, x) \leq C(1+|x|) \delta^{\frac{1}{2}}
$$

From it we obtain immediately that $W$ is $\frac{1}{2}-$ Hölder continuous in $t$. We will only check the second inequality in (4.30), the first one can be shown in a similar way. To this end we note that due to (4.28), for an arbitrarily small $\varepsilon>0$,

$$
W(t, x)-W(t+\delta, x) \leq I_{\delta}^{1}+I_{\delta}^{2}+\varepsilon,
$$

where

$$
\begin{aligned}
I_{\delta}^{1} & :=G_{t, t+\delta}^{t, x ; u^{\varepsilon}, \beta\left(u^{\varepsilon}\right)}\left[W\left(t+\delta, X_{t+\delta}^{t, x ; u^{\varepsilon}, \beta\left(u^{\varepsilon}\right)}\right)\right]-G_{t, t+\delta}^{t, x ; u^{\varepsilon}, \beta\left(u^{\varepsilon}\right)}[W(t+\delta, x)], \\
I_{\delta}^{2} & :=G_{t, t+\delta}^{t, x ; u^{\varepsilon}, \beta\left(u^{\varepsilon}\right)}[W(t+\delta, x)]-W(t+\delta, x),
\end{aligned}
$$


for arbitrarily chosen $\beta \in \mathcal{B}_{t, t+\delta}$ and $u^{\varepsilon} \in \mathcal{U}_{t, t+\delta}$ such that (4.28) holds. From Lemma 2.3 and the estimate (4.12) we obtain that, for some constant $C$ independent of the controls $u^{\varepsilon}$ and $\beta\left(u^{\varepsilon}\right)$,

$$
\begin{aligned}
\left|I_{\delta}^{1}\right| & \leq\left[C E\left(\left|W\left(t+\delta, X_{t+\delta}^{t, x ; u^{\varepsilon}, \beta\left(u^{\varepsilon}\right)}\right)-W(t+\delta, x)\right|^{2} \mid \mathcal{F}_{t}\right)\right]^{\frac{1}{2}} \\
& \leq\left[C E\left(\left|X_{t+\delta}^{t, x ; u^{\varepsilon}, \beta\left(u^{\varepsilon}\right)}-x\right|^{2} \mid \mathcal{F}_{t}\right)\right]^{\frac{1}{2}}
\end{aligned}
$$

and since $E\left[\left|X_{t+\delta}^{t, x ; u^{\varepsilon}, \beta\left(u^{\varepsilon}\right)}-x\right|^{2} \mid \mathcal{F}_{t}\right] \leq C\left(1+|x|^{2}\right) \delta$ we deduce that $\left|I_{\delta}^{1}\right| \leq C(1+|x|) \delta^{\frac{1}{2}}$. From the definition of $G_{t, t+\delta}^{t, x ; u^{\varepsilon}, \beta\left(u^{\varepsilon}\right)}[\cdot]$ (see (4.13)) we know that the second term $I_{\delta}^{2}$ can be written as

$$
\begin{aligned}
I_{\delta}^{2}= & E\left[W(t+\delta, x)+\int_{t}^{t+\delta} f\left(s, X_{s}^{t, x ; u^{\varepsilon}, \beta\left(u^{\varepsilon}\right)}, \tilde{Y}_{s}^{t, x ; u^{\varepsilon}, \beta\left(u^{\varepsilon}\right)}, \tilde{Z}_{s}^{t, x ; u^{\varepsilon}, \beta\left(u^{\varepsilon}\right)}, u_{s}^{\varepsilon}, \beta_{s}\left(u_{.}^{\varepsilon}\right)\right) d s\right. \\
& \left.-\int_{t}^{t+\delta} \tilde{Z}_{s}^{t, x ; u^{\varepsilon}, \beta\left(u^{\varepsilon}\right)} d B_{s} \mid \mathcal{F}_{t}\right]-W(t+\delta, x) \\
= & E\left[\int_{t}^{t+\delta} f\left(s, X_{s}^{t, x ; u^{\varepsilon}, \beta\left(u^{\varepsilon}\right)}, \tilde{Y}_{s}^{t, x ; u^{\varepsilon}, \beta\left(u^{\varepsilon}\right)}, \tilde{Z}_{s}^{t, x ; u^{\varepsilon}, \beta\left(u^{\varepsilon}\right)}, u_{s}^{\varepsilon}, \beta_{s}\left(u^{\varepsilon}\right)\right) d s \mid \mathcal{F}_{t}\right] .
\end{aligned}
$$

With the help of the Schwartz inequality, the estimates (4.3) and (3.4)-(i), we then have

$$
\begin{aligned}
\left|I_{\delta}^{2}\right| & \leq \delta^{\frac{1}{2}} E\left[\int_{t}^{t+\delta}\left|f\left(s, X_{s}^{t, x ; u^{\varepsilon}, \beta\left(u^{\varepsilon}\right)}, \tilde{Y}_{s}^{t, x ; u^{\varepsilon}, \beta\left(u^{\varepsilon}\right)}, \tilde{Z}_{s}^{t, x ; u^{\varepsilon}, \beta\left(u^{\varepsilon}\right)}, u_{s}^{\varepsilon}, \beta_{s}\left(u^{\varepsilon}\right)\right)\right|^{2} d s \mid \mathcal{F}_{t}\right]^{\frac{1}{2}} \\
& \leq \delta^{\frac{1}{2}} E\left[\int_{t}^{t+\delta}\left(\left|f\left(s, X_{s}^{t, x ; u^{\varepsilon}, \beta\left(u^{\varepsilon}\right)}, 0,0, u_{s}^{\varepsilon}, \beta_{s}\left(u^{\varepsilon}\right)\right)\right|+C\left|\tilde{Y}_{s}^{t, x ; u^{\varepsilon}, \beta\left(u^{\varepsilon}\right)}\right|+C\left|\tilde{Z}_{s}^{t, x ; u^{\varepsilon}, \beta\left(u^{\varepsilon}\right)}\right|\right)^{2} d s \mid \mathcal{F}_{t}\right]^{\frac{1}{2}} \\
& \leq C \delta^{\frac{1}{2}} E\left[\int_{t}^{t+\delta}\left(|1+| X_{s}^{t, x ; u^{\varepsilon}, \beta\left(u^{\varepsilon}\right)}|+| \tilde{Y}_{s}^{t, x ; u^{\varepsilon}, \beta\left(u^{\varepsilon}\right)}|+| \tilde{Z}_{s}^{t, x ; u^{\varepsilon}, \beta\left(u^{\varepsilon}\right)} \mid\right)^{2} d s \mid \mathcal{F}_{t}\right]^{\frac{1}{2}} \\
& \leq C(1+|x|) \delta^{\frac{1}{2}} .
\end{aligned}
$$

Hence, from (4.31),

$$
W(t, x)-W(t+\delta, x) \leq C(1+|x|) \delta^{\frac{1}{2}}+\varepsilon,
$$

and letting $\varepsilon \downarrow 0$ we get the second inequality of (4.30). The proof is complete.

\section{Viscosity Solution of Isaacs' Equation: Existence Theorem}

In this section we consider the following Isaacs' equations

$$
\left\{\begin{array}{l}
\frac{\partial}{\partial t} W(t, x)+H^{-}\left(t, x, W, D W, D^{2} W\right)=0, \quad(t, x) \in[0, T) \times \mathbb{R}^{n}, \\
W(T, x)=\Phi(x), \quad x \in \mathbb{R}^{n},
\end{array}\right.
$$

and

$$
\left\{\begin{array}{l}
\frac{\partial}{\partial t} U(t, x)+H^{+}\left(t, x, U, D U, D^{2} U\right)=0, \quad(t, x) \in[0, T) \times \mathbb{R}^{n}, \\
U(T, x)=\Phi(x), \quad x \in \mathbb{R}^{n},
\end{array}\right.
$$

associated with the Hamiltonians

$$
H^{-}(t, x, y, p, X)=\sup _{u \in U} \inf _{v \in V}\left\{\frac{1}{2} \operatorname{tr}\left(\sigma \sigma^{T}(t, x, u, v) X\right)+p . b(t, x, u, v)+f(t, x, y, p . \sigma, u, v)\right\}
$$

and

$$
H^{+}(t, x, y, p, X)=\inf _{v \in V} \sup _{u \in U}\left\{\frac{1}{2} \operatorname{tr}\left(\sigma \sigma^{T}(t, x, u, v) X\right)+p . b(t, x, u, v)+f(t, x, y, p . \sigma, u, v)\right\},
$$

respectively, where $t \in[0, T], x \in \mathbb{R}^{n}, y \in \mathbb{R}, p \in \mathbb{R}^{n}$ and $X \in \mathbf{S}^{n}$ (recall that $\mathbf{S}^{n}$ denotes the set of $n \times n$ symmetric matrices). Here the functions $b, \sigma, f$ and $\Phi$ are supposed to satisfy (H4.1) and (H4.2), respectively. 
In this section we want to prove that the lower value function $W(t, x)$ introduced by (4.9) is the viscosity solution of equation (5.1), while the upper value function $U(t, x)$ defined by (4.10) is the viscosity solution of equation (5.2). For this we translate Peng's BSDE approach [14] developed in the framework of stochastic control theory into that of the stochastic differential games. Uniqueness of the viscosity solution will be shown in the next section for the class of continuous functions satisfying some growth assumption which is weaker than the polynomial growth condition. We first recall the definition of a viscosity solution of equation (5.1), similarly for equation (5.2). The reader more interested in viscosity solutions is referred to Crandall, Ishii and Lions [5].

Definition 5.1. A real-valued continuous function $W \in C\left([0, T] \times \mathbb{R}^{n}\right)$ is called (i) a viscosity subsolution of equation (5.1) if $W(T, x) \leq \Phi(x)$, for all $x \in \mathbb{R}^{n}$, and if for all functions $\varphi \in C_{l, b}^{3}\left([0, T] \times \mathbb{R}^{n}\right)$ and $(t, x) \in[0, T) \times \mathbb{R}^{n}$ such that $W-\varphi$ attains its local maximum at $(t, x)$,

$$
\frac{\partial \varphi}{\partial t}(t, x)+H^{-}\left(t, x, \varphi, D \varphi, D^{2} \varphi\right) \geq 0
$$

(ii) a viscosity supersolution of equation (5.1) if $W(T, x) \geq \Phi(x)$, for all $x \in \mathbb{R}^{n}$, and if for all functions $\varphi \in C_{l, b}^{3}\left([0, T] \times \mathbb{R}^{n}\right)$ and $(t, x) \in[0, T) \times \mathbb{R}^{n}$ such that $W-\varphi$ attains its local minimum at $(t, x)$,

$$
\frac{\partial \varphi}{\partial t}(t, x)+H^{-}\left(t, x, \varphi, D \varphi, D^{2} \varphi\right) \leq 0 ;
$$

(iii) a viscosity solution of equation (5.1) if it is both a viscosity sub- and a supersolution of equation (5.1).

Remark 5.1. $C_{l, b}^{3}\left([0, T] \times \mathbb{R}^{n}\right)$ denotes the set of the real-valued functions that are continuously differentiable up to the third order and whose derivatives of order from 1 to 3 are bounded.

We first prove that the lower value function $W(t, x)$ is a viscosity solution of equation (5.1).

Theorem 5.1. Under the assumptions (H4.1) and (H4.2) the lower value function $W(t, x)$ is a viscosity solution of equation (5.1).

For the proof of this theorem we need four auxiliary lemmata. To abbreviate notation we put, for some arbitrarily chosen but fixed $\varphi \in C_{l, b}^{3}\left([0, T] \times \mathbb{R}^{n}\right)$,

$$
\begin{array}{r}
F(s, x, y, z, u, v)=\frac{\partial}{\partial s} \varphi(s, x)+\frac{1}{2} \operatorname{tr}\left(\sigma \sigma^{T}(s, x, u, v) D^{2} \varphi\right)+D \varphi \cdot b(s, x, u, v) \\
+f(s, x, y+\varphi(s, x), z+D \varphi(s, x) \cdot \sigma(s, x, u, v), u, v)
\end{array}
$$

$(s, x, y, z, u, v) \in[0, T] \times \mathbb{R}^{n} \times \mathbb{R} \times \mathbb{R}^{d} \times U \times V$, and we consider the following BSDE defined on the interval $[t, t+\delta](0<\delta \leq T-t)$ :

$$
\left\{\begin{aligned}
-d Y_{s}^{1, u, v} & =F\left(s, X_{s}^{t, x ; u, v}, Y_{s}^{1, u, v}, Z_{s}^{1, u, v}, u_{s}, v_{s}\right) d s-Z_{s}^{1, u, v} d B_{s}, \\
Y_{t+\delta}^{1, u, v} & =0
\end{aligned}\right.
$$

where the process $X^{t, x, u, v}$ has been introduced by equation $(4.1)$ and $u(\cdot) \in \mathcal{U}_{t, t+\delta}, v(\cdot) \in \mathcal{V}_{t, t+\delta}$.

Remark 5.2. It's not hard to check that $F\left(s, X_{s}^{t, x ; u, v}, y, z, u_{s}, v_{s}\right)$ satisfies (A1) and (A2). Thus, due to Lemma 2.1 equation (5.4) has a unique solution. 
We can characterize the solution process $Y^{1, u, v}$ as follows:

Lemma 5.1. For every $s \in[t, t+\delta]$, we have the following relationship:

$$
Y_{s}^{1, u, v}=G_{s, t+\delta}^{t, x ; u, v}\left[\varphi\left(t+\delta, X_{t+\delta}^{t, x ; u, v}\right)\right]-\varphi\left(s, X_{s}^{t, x ; u, v}\right), \quad P-a . s . .
$$

Proof: We recall that $G_{s, t+\delta}^{t, x ; u}\left[\varphi\left(t+\delta, X_{t+\delta}^{t, x ; u, v}\right)\right]$ is defined with the help of the solution of the BSDE

$$
\left\{\begin{aligned}
-d Y_{s}^{u, v} & =f\left(s, X_{s}^{t, x ; u, v}, Y_{s}^{u, v}, Z_{s}^{u, v}, u_{s}, v_{s}\right) d s-Z_{s}^{u, v} d B_{s}, \quad s \in[t, t+\delta], \\
Y_{t+\delta}^{u, v} & =\varphi\left(t+\delta, X_{t+\delta}^{t, x ; u, v}\right),
\end{aligned}\right.
$$

by the following formula:

$$
G_{s, t+\delta}^{t, x ; u, v}\left[\varphi\left(t+\delta, X_{t+\delta}^{t, x ; u, v}\right)\right]=Y_{s}^{u, v}, \quad s \in[t, t+\delta]
$$

(see (4.13)). Therefore we only need to prove that $Y_{s}^{u, v}-\varphi\left(s, X_{s}^{t, x ; u, v}\right) \equiv Y_{s}^{1, u, v}$. This result can be obtained easily by applying Itô's formula to $\varphi\left(s, X_{s}^{t, x ; u, v}\right)$. Indeed, we get that the stochastic differentials of $Y_{s}^{u, v}-\varphi\left(s, X_{s}^{t, x ; u, v}\right)$ and $Y_{s}^{1, u, v}$ coincide, while at the terminal time $t+\delta, Y_{t+\delta}^{u, v}-$ $\varphi\left(t+\delta, X_{t+\delta}^{t, x ; u, v}\right)=0=Y_{t+\delta}^{1, u, v}$. So the proof is complete.

Now we consider the following simple BSDE in which the driving process $X^{t, x ; u, v}$ is replaced by its deterministic initial value $x$ :

$$
\left\{\begin{aligned}
-d Y_{s}^{2, u, v} & =F\left(s, x, Y_{s}^{2, u, v}, Z_{s}^{2, u, v}, u_{s}, v_{s}\right) d s-Z_{s}^{2, u, v} d B_{s} \\
Y_{t+\delta}^{2, u, v} & =0, \quad s \in[t, t+\delta]
\end{aligned}\right.
$$

where $u(\cdot) \in \mathcal{U}_{t, t+\delta}, v(\cdot) \in \mathcal{V}_{t, t+\delta}$. The following Lemma will allow us to neglect the difference $\left|Y_{t}^{1, u, v}-Y_{t}^{2, u, v}\right|$ for sufficiently small $\delta>0$.

Lemma 5.2. For every $u \in \mathcal{U}_{t, t+\delta}, v \in \mathcal{V}_{t, t+\delta}$, we have

$$
\left|Y_{t}^{1, u, v}-Y_{t}^{2, u, v}\right| \leq C \delta^{\frac{3}{2}}, \quad P-a . s .,
$$

where $C$ is independent of the control processes $u$ and $v$.

Proof: From (4.3) we have for all $p \geq 2$ the existence of some $C_{p} \in \mathbb{R}_{+}$such that

$$
E\left[\sup _{t \leq s \leq T}\left|X_{s}^{t, x ; u, v}\right|^{p} \mid \mathcal{F}_{t}\right] \leq C_{p}\left(1+|x|^{p}\right), \quad \text { P-a.s., uniformly in } u \in \mathcal{U}_{t, t+\delta}, v \in \mathcal{V}_{t, t+\delta}
$$

This combined with the estimate

$$
\begin{aligned}
E\left[\sup _{t \leq s \leq t+\delta}\left|X_{s}^{t, x ; u, v}-x\right|^{p} \mid \mathcal{F}_{t}\right] \leq & 2^{p-1} E\left[\sup _{t \leq s \leq t+\delta}\left|\int_{t}^{s} b\left(r, X_{r}^{t, x ; u, v}, u_{r}, v_{r}\right) d r\right|^{p} \mid \mathcal{F}_{t}\right] \\
& +2^{p-1} E\left[\sup _{t \leq s \leq t+\delta}\left|\int_{t}^{s} \sigma\left(r, X_{r}^{t, x ; u, v}, u_{r}, v_{r}\right) d B_{r}\right|^{p} \mid \mathcal{F}_{t}\right]
\end{aligned}
$$

yields

$$
E\left[\sup _{t \leq s \leq t+\delta}\left|X_{s}^{t, x ; u, v}-x\right|^{p} \mid \mathcal{F}_{t}\right] \leq C_{p} \delta^{\frac{p}{2}}, \quad \text { P-a.s., } \quad \text { uniformly in } u \in \mathcal{U}_{t, t+\delta}, v \in \mathcal{V}_{t, t+\delta}
$$


We now apply Lemma 2.3 combined with (5.9) to equations (5.4) and (5.7). For this we set in Lemma 2.3:

$$
\begin{gathered}
\xi_{1}=\xi_{2}=0, g(s, y, z)=F\left(s, X_{s}^{t, x, u, v}, y, z, u_{s}, v_{s}\right) \\
\varphi_{1}(s)=0, \varphi_{2}(s)=F\left(s, x, Y_{s}^{2, u, v}, Z_{s}^{2, u, v}, u_{s}, v_{s}\right)-F\left(s, X_{s}^{t, x, u, v}, Y_{s}^{2, u, v}, Z_{s}^{2, u, v}, u_{s}, v_{s}\right) .
\end{gathered}
$$

Obviously, the function $g$ is Lipschitz with respect to $(y, z)$, and $\left|\varphi_{2}(s)\right| \leq C\left(1+|x|^{2}\right)\left(\mid X_{s}^{t, x ; u, v}-\right.$ $\left.x|+| X_{s}^{t, x ; u, v}-\left.x\right|^{3}\right)$, for $s \in[t, t+\delta],(t, x) \in[0, T) \times \mathbb{R}^{n}, u \in \mathcal{U}_{t, t+\delta}, v \in \mathcal{V}_{t, t+\delta}$. Thus, with the notation $\rho_{0}(r)=\left(1+|x|^{2}\right)\left(r+r^{3}\right), r \geq 0$, we have

$$
\begin{aligned}
& E\left[\int_{t}^{t+\delta}\left(\left|Y_{s}^{1, u, v}-Y_{s}^{2, u, v}\right|^{2}+\left|Z_{s}^{1, u, v}-Z_{s}^{2, u, v}\right|^{2}\right) d s \mid \mathcal{F}_{t}\right] \\
\leq & C E\left[\int_{t}^{t+\delta} \rho_{0}^{2}\left(\left|X_{s}^{t, x, u, v}-x\right|\right) d s \mid \mathcal{F}_{t}\right] \\
\leq & C \delta E\left[\sup _{t \leq s \leq t+\delta} \rho_{0}^{2}\left(\left|X_{s}^{t, x, u, v}-x\right|\right) \mid \mathcal{F}_{t}\right] \\
\leq & C \delta^{2} .
\end{aligned}
$$

Therefore,

$$
\begin{aligned}
& \left|Y_{t}^{1, u, v}-Y_{t}^{2, u, v}\right|=\left|E\left[\left(Y_{t}^{1, u, v}-Y_{t}^{2, u, v}\right) \mid \mathcal{F}_{t}\right]\right| \\
= & \left|E\left[\int_{t}^{t+\delta}\left(F\left(s, X_{s}^{t, x, u, v}, Y_{s}^{1, u, v}, Z_{s}^{1, u, v}, u_{s}, v_{s}\right)-F\left(s, x, Y_{s}^{2, u, v}, Z_{s}^{2, u, v}, u_{s}, v_{s}\right)\right) d s \mid \mathcal{F}_{t}\right]\right| \\
\leq & C E\left[\int_{t}^{t+\delta}\left[\rho_{0}\left(\left|X_{s}^{t, x, u, v}-x\right|\right)+\left|Y_{s}^{1, u, v}-Y_{s}^{2, u, v}\right|+\left|Z_{s}^{1, u, v}-Z_{s}^{2, u, v}\right|\right] d s \mid \mathcal{F}_{t}\right] \\
\leq & C E\left[\int_{t}^{t+\delta} \rho_{0}\left(\left|X_{s}^{t, x, u, v}-x\right|\right) d s \mid \mathcal{F}_{t}\right]+C \delta^{\frac{1}{2}}\left\{E\left[\int_{t}^{t+\delta}\left|Y_{s}^{1, u, v}-Y_{s}^{2, u, v}\right|^{2} \mid \mathcal{F}_{t}\right]^{\frac{1}{2}}\right. \\
& \left.+E\left[\int_{t}^{t+\delta}\left|Z_{s}^{1, u, v}-Z_{s}^{2, u, v}\right|^{2} d s \mid \mathcal{F}_{t}\right]^{\frac{1}{2}}\right\} \\
\leq & C \delta^{\frac{3}{2}}
\end{aligned}
$$

Thus, the proof is complete.

Lemma 5.3. Let $Y_{0}(\cdot)$ be the solution of the following ordinary differential equation:

$$
\left\{\begin{array}{l}
-\dot{Y}_{0}(s)=F_{0}\left(s, x, Y_{0}(s), 0\right), \quad s \in[t, t+\delta] \\
Y_{0}(t+\delta)=0
\end{array}\right.
$$

where the function $F_{0}$ is defined by

$$
F_{0}(s, x, y, z)=\sup _{u \in U} \inf _{v \in V} F(s, x, y, z, u, v) .
$$

Then, P-a.s.,

$$
\operatorname{essup}_{u \in \mathcal{U}_{t, t+\delta}} \operatorname{essinf}_{v \in \mathcal{V}_{t, t+\delta}} Y_{t}^{2, u, v}=Y_{0}(t)
$$

Proof: Obviously, $F_{0}(s, x, y, z)$ is Lipschitz in $(y, z)$, uniformly with respect to $(s, x)$. This guarantees existence and uniqueness for equation (5.10). We first introduce the function

$$
F_{1}(s, x, y, z, u)=\inf _{v \in V} F(s, x, y, z, u, v),(s, x, y, z, u) \in[0, T] \times \mathbb{R}^{n} \times \mathbb{R} \times \mathbb{R}^{d} \times U,
$$

and consider the BSDE

$$
\left\{\begin{array}{l}
-d Y^{3, u}(s)=F_{1}\left(s, x, Y^{3, u}(s), Z^{3, u}(s), u_{s}\right) d s-Z^{3, u}(s) d B_{s} \\
Y^{3, u}(t+\delta)=0, \quad s \in[t, t+\delta]
\end{array}\right.
$$


for $u \in \mathcal{U}_{t, t+\delta}$. We notice that since $F_{1}\left(s, x, y, z, u_{s}\right)$ is Lipschitz in $(y, z)$, for every $u \in \mathcal{U}_{t, t+\delta}$, there exists a unique solution $\left(Y^{3, u}, Z^{3, u}\right)$ to the BSDE (5.14). Moreover,

$$
Y^{3, u}(t)=\operatorname{essinf}_{v(\cdot) \in \mathcal{V}_{t, t+\delta}} Y_{t}^{2, u, v}, \text { P-a.s., for any } u \in \mathcal{U}_{t, t+\delta} .
$$

Indeed, from the definition of $F_{1}$ and Lemma 2.2 (comparison theorem) we have

$$
Y^{3, u}(t) \leq \operatorname{essinf}_{v(\cdot) \in \mathcal{V}_{t, t+\delta}} Y_{t}^{2, u, v}, \text { P-a.s., for all } u \in \mathcal{U}_{t, t+\delta} .
$$

On the other hand, there exists a measurable function $v^{3}:[t, T] \times \mathbb{R}^{n} \times \mathbb{R} \times \mathbb{R}^{d} \times U \rightarrow V$ such that

$$
F_{1}(s, x, y, z, u)=F\left(s, x, y, z, u, v^{3}(s, x, y, z, u)\right) \text {, for any } s, x, y, z, u \text {. }
$$

We then put

$$
\widetilde{v}_{s}^{3}:=v^{3}\left(s, x, Y_{s}^{3, u}, Z_{s}^{3, u}, u_{s}\right), s \in[t, t+\delta],
$$

and we observe that $\widetilde{v}^{3} \in \mathcal{V}_{t, t+\delta}$, and

$$
F_{1}\left(s, x, Y_{s}^{3, u}, Z_{s}^{3, u}, u_{s}\right)=F\left(s, x, Y_{s}^{3, u}, Z_{s}^{3, u}, u_{s}, \widetilde{v}_{s}^{3}\right), s \in[t, t+\delta] .
$$

Consequently, from the uniqueness of the solution of the BSDE it follows that $\left(Y^{3, u}, Z^{3, u}\right)=$ $\left(Y^{2, u, \widetilde{v}^{3}}, Z^{2, u, \widetilde{v}^{3}}\right)$ and, in particular, $Y_{t}^{3, u}=Y_{t}^{2, u, \widetilde{v}^{3}}$, P-a.s., for any $u \in \mathcal{U}_{t, t+\delta}$. This proves that

$$
Y^{3, u}(t)=\operatorname{essinf}_{v \in \mathcal{V}_{t, t+\delta}} Y_{t}^{2, u, v}, \text { P-a.s., for all } u \in \mathcal{U}_{t, t+\delta} .
$$

Finally, since $F_{0}(s, x, y, z)=\sup _{u \in U} F_{1}(s, x, y, z, u)$, an argument similar to that developed above yields

$$
Y_{0}(t)=\operatorname{esssup}_{u \in \mathcal{U}_{t, t+\delta}} Y^{3, u}(t)\left(=\operatorname{esssup}_{u \in \mathcal{U}_{t, t+\delta}} \operatorname{essinf}_{v \in \mathcal{V}_{t, t+\delta}} Y_{t}^{2, u, v}\right), \text { P-a.s. }
$$

It uses the fact that equation (5.10) can be considered as a BSDE with solution $\left(Y_{s}, Z_{s}\right)=\left(Y_{0}(s), 0\right)$. The proof is complete.

Lemma 5.4. For every $u \in \mathcal{U}_{t, t+\delta}, v \in \mathcal{V}_{t, t+\delta}$, we have

$$
E\left[\int_{t}^{t+\delta}\left|Y_{s}^{2, u, v}\right| d s \mid \mathcal{F}_{t}\right]+E\left[\int_{t}^{t+\delta}\left|Z_{s}^{2, u, v}\right| d s \mid \mathcal{F}_{t}\right] \leq C \delta^{\frac{3}{2}}, \quad P-a . s .,
$$

where the constant $C$ is independent of the controls $u, v$.

Proof: Since $F(s, x, \cdot, \cdot, u, v)$ has a linear growth in $(y, z)$, uniformly in $(u, v)$, we get from Lemma 2.3 that, for some constant $C$ independent of $\delta$ and the control processes $u, v$,

$$
\left|Y_{s}^{2, u, v}\right|^{2} \leq C \delta, E\left[\int_{s}^{t+\delta}\left|Z_{r}^{2, u, v}\right|^{2} d r \mid \mathcal{F}_{s}\right] \leq C \delta, s \in[t, t+\delta] .
$$

On the other hand, from equation (5.7),

$$
\begin{aligned}
\left|Y_{s}^{2, u, v}\right| & \leq E\left[\int_{s}^{t+\delta}\left|F\left(r, x, Y_{r}^{2, u, v}, Z_{r}^{2, u, v}, u_{r}, v_{r}\right)\right| d r \mid \mathcal{F}_{s}\right] \\
& \leq C E\left[\int_{s}^{t+\delta}\left(1+|x|^{2}+\left|Y_{r}^{2, u, v}\right|+\left|Z_{r}^{2, u, v}\right|\right) d r \mid \mathcal{F}_{s}\right] \\
& \leq C \delta+C \sqrt{\delta}\left(E\left[\int_{s}^{t+\delta}\left|Z_{r}^{2, u, v}\right|^{2} d r \mid \mathcal{F}_{s}\right]\right)^{\frac{1}{2}} \leq C \delta, \text { P-a.s., } s \in[t, t+\delta],
\end{aligned}
$$


and, since

$$
\int_{t}^{t+\delta} Z_{s}^{2, u, v} d B_{s}=\int_{t}^{t+\delta} F\left(s, x, Y_{s}^{2, u, v}, Z_{s}^{2, u, v}, u_{s}, v_{s}\right) d s-Y_{t}^{2, u, v},
$$

we can get $E\left[\int_{t}^{t+\delta}\left|Z_{s}^{2, u, v}\right|^{2} d s \mid \mathcal{F}_{t}\right] \leq C \delta^{2}$. Finally,

$$
\begin{aligned}
& E\left[\int_{t}^{t+\delta}\left|Y_{s}^{2, u, v}\right| d s \mid \mathcal{F}_{t}\right]+E\left[\int_{t}^{t+\delta}\left|Z_{s}^{2, u, v}\right| d s \mid \mathcal{F}_{t}\right] \leq C \delta^{2}+\delta^{\frac{1}{2}}\left\{E\left[\int_{t}^{t+\delta}\left|Z_{s}^{2, u, v}\right|^{2} d s \mid \mathcal{F}_{t}\right]\right\}^{\frac{1}{2}} \\
& \leq C \delta^{\frac{3}{2}}
\end{aligned}
$$

The proof is complete.

Now we are able to give the proof of Theorem 5.1:

Proof: (1) Obviously, $W(T, x)=\Phi(x), x \in \mathbb{R}^{n}$. Let us show in a first step that $W$ is a viscosity supersolution. For this we suppose that $\varphi \in C_{l, b}^{3}\left([0, T] \times \mathbb{R}^{n}\right)$, and $(t, x) \in[0, T) \times \mathbb{R}^{n}$ are such that $W-\varphi$ attains its minimum at $(t, x)$. Notice that we can replace the condition of a local minimum by that of a global one in the definition of the viscosity supersolution since $W$ is continuous and of at most linear growth. Without loss of generality we may also suppose that $\varphi(t, x)=W(t, x)$. Then, due to the DPP (see Theorem 4.2),

$$
\varphi(t, x)=W(t, x)=\operatorname{essinf}_{\beta \in \mathcal{B}_{t, t+\delta}} \operatorname{esssup}_{u \in \mathcal{U}_{t, t+\delta}} G_{t, t+\delta}^{t, x ; u, \beta(u)}\left[W\left(t+\delta, X_{t+\delta}^{t, x ; u, \beta(u)}\right)\right], 0 \leq \delta \leq T-t,
$$

and from $W \geq \varphi$ and the monotonicity property of $G_{t, t+\delta}^{t, x ; u, \beta(u)}[\cdot]$ (see Lemma 2.2) we obtain

$$
\operatorname{essinf}_{\beta \in \mathcal{B}_{t, t+\delta}} \operatorname{esssup}_{u \in \mathcal{U}_{t, t+\delta}}\left\{G_{t, t+\delta}^{t, x ; u, \beta(u)}\left[\varphi\left(t+\delta, X_{t+\delta}^{t, x ; u, \beta(u)}\right)\right]-\varphi(t, x)\right\} \leq 0, \text { P-a.s.. }
$$

Thus, from Lemma 5.1,

$$
\operatorname{essinf}_{\beta \in \mathcal{B}_{t, t+\delta}} \operatorname{esssup}_{u \in \mathcal{U}_{t, t+\delta}} Y_{t}^{1, u, \beta(u)} \leq 0 \text {, P-a.s., }
$$

and further, from Lemma 5.2 we have

$$
\operatorname{essinf}_{\beta \in \mathcal{B}_{t, t+\delta}} \operatorname{esssup}_{u \in \mathcal{U}_{t, t+\delta}} Y_{t}^{2, u, \beta(u)} \leq C \delta^{\frac{3}{2}}, \text { P-a.s.. }
$$

Consequently, since $\operatorname{essinf}_{v \in \mathcal{V}_{t, t+\delta}} Y_{t}^{2, u, v} \leq Y_{t}^{2, u, \beta(u)}, \beta \in \mathcal{B}_{t, t+\delta}$, we get

$$
\operatorname{esssup}_{u \in \mathcal{U}_{t, t+\delta}} \operatorname{essinf}_{v \in \mathcal{V}_{t, t+\delta}} Y_{t}^{2, u, v} \leq \operatorname{essinf}_{\beta \in \mathcal{B}_{t, t+\delta}} \operatorname{esssup}_{u \in \mathcal{U}_{t, t+\delta}} Y_{t}^{2, u, \beta(u)} \leq C \delta^{\frac{3}{2}}, \text { P-a.s., }
$$

and Lemma 5.3 implies

$$
Y_{0}(t) \leq C \delta^{\frac{3}{2}}, \text { P-a.s., }
$$

where $Y_{0}$ is the unique solution of equation (5.10). It then follows easily that

$$
\sup _{u \in U} \inf _{v \in V} F(t, x, 0,0, u, v)=F_{0}(t, x, 0,0) \leq 0,
$$

and from the definition of $F$ we see that $W$ is a viscosity supersolution of equation (5.1).

(2) The second step is devoted to the proof that $W$ is a viscosity subsolution. For this we suppose that $\varphi \in C_{l, b}^{3}\left([0, T] \times \mathbb{R}^{n}\right)$ and $(t, x) \in[0, T) \times \mathbb{R}^{n}$ are such that $W-\varphi$ attains its maximum at $(t, x)$. Without loss of generality we suppose again $\varphi(t, x)=W(t, x)$. We must prove that

$$
\sup _{u \in U} \inf _{v \in V} F(t, x, 0,0, u, v)=F_{0}(t, x, 0,0) \geq 0 .
$$


Let us suppose that this is not true. Then there exists some $\theta>0$ such that

$$
F_{0}(t, x, 0,0)=\sup _{u \in U} \inf _{v \in V} F(t, x, 0,0, u, v) \leq-\theta<0,
$$

and we can find a measurable function $\psi: U \rightarrow V$ such that

$$
F(t, x, 0,0, u, \psi(u)) \leq-\frac{3}{4} \theta, \text { for all } u \in U .
$$

Moreover, since $F(\cdot, x, 0,0, \cdot, \cdot)$ is uniformly continuous on $[0, T] \times U \times V$ there exists some $T-t \geq$ $R>0$ such that

$$
F(s, x, 0,0, u, \psi(u)) \leq-\frac{1}{2} \theta, \text { for all } u \in U \text { and }|s-t| \leq R .
$$

On the other hand, due to the DPP (see Theorem 4.1), for every $\delta \in(0, R]$,

$$
\varphi(t, x)=W(t, x)=\operatorname{essinf}_{\beta \in \mathcal{B}_{t, t+\delta}} \operatorname{esssup}_{u \in \mathcal{U}_{t, t+\delta}} G_{t, t+\delta}^{t, x ; u, \beta(u)}\left[W\left(t+\delta, X_{t+\delta}^{t, x ; u, \beta(u)}\right)\right],
$$

and from $W \leq \varphi$ and the monotonicity property of $G_{t, t+\delta}^{t, x ; u, \beta(u)}[\cdot]$ (see Lemma 2.2) we obtain

$$
\operatorname{essinf}_{\beta \in \mathcal{B}_{t, t+\delta}} \operatorname{esssup}_{u \in \mathcal{U}_{t, t+\delta}}\left\{G_{t, t+\delta}^{t, x ; u, \beta(u)}\left[\varphi\left(t+\delta, X_{t+\delta}^{t, x ; u, \beta(u)}\right)\right]-\varphi(t, x)\right\} \geq 0, \text { P-a.s.. }
$$

Thus, from Lemma 5.1,

$$
\operatorname{essinf}_{\beta \in \mathcal{B}_{t, t+\delta}} \operatorname{esssup}_{u \in \mathcal{U}_{t, t+\delta}} Y_{t}^{1, u, \beta(u)} \geq 0, \text { P-a.s. }
$$

and, in particular,

$$
\operatorname{esssup}_{u \in \mathcal{U}_{t, t+\delta}} Y_{t}^{1, u, \psi(u)} \geq 0, \text { P-a.s.. }
$$

Here, by putting $\psi_{s}(u)(\omega)=\psi\left(u_{s}(\omega)\right),(s, \omega) \in[t, T] \times \Omega$, we identify $\psi$ as an element of $\mathcal{B}_{t, t+\delta}$. Given an arbitrarily $\varepsilon>0$ we can choose $u^{\varepsilon} \in \mathcal{U}_{t, t+\delta}$ such that $Y_{t}^{1, u^{\varepsilon}, \psi\left(u^{\varepsilon}\right)} \geq-\varepsilon \delta$. For this the argument developed in the proof of the lemmata can be used. From Lemma 5.2 we further have

$$
Y_{t}^{2, u^{\varepsilon}, \psi\left(u^{\varepsilon}\right)} \geq-C \delta^{\frac{3}{2}}-\varepsilon \delta, \text { P-a.s.. }
$$

Taking into account that $Y_{t}^{2, u^{\varepsilon}, \psi\left(u^{\varepsilon}\right)}=E\left[\int_{t}^{t+\delta} F\left(s, x, Y_{s}^{2, u^{\varepsilon}, \psi\left(u^{\varepsilon}\right)}, Z_{s}^{2, u^{\varepsilon}, \psi\left(u^{\varepsilon}\right)}, u_{s}^{\varepsilon}, \psi_{s}\left(u^{\varepsilon}\right)\right) d s \mid \mathcal{F}_{t}\right]$ we get from the Lipschitz property of $F$ in $(y, z),(5.17)$ and Lemma 5.4 that

$$
\begin{aligned}
Y_{t}^{2, u^{\varepsilon}, \psi\left(u^{\varepsilon}\right)} & \leq E\left[\int_{t}^{t+\delta}\left(C\left|Y_{s}^{2, u^{\varepsilon}, \psi\left(u^{\varepsilon}\right)}\right|+C\left|Z_{s}^{2, u^{\varepsilon}, \psi\left(u^{\varepsilon}\right)}\right|+F\left(s, x, 0,0, u_{s}^{\varepsilon}, \psi_{s}\left(u_{.}^{\varepsilon}\right)\right)\right) d s \mid \mathcal{F}_{t}\right] \\
& \leq C \delta^{\frac{3}{2}}-\frac{1}{2} \theta \delta, \text { P-a.s.. }
\end{aligned}
$$

From (5.18) and (5.19), $-C \delta^{\frac{1}{2}}-\varepsilon \leq C \delta^{\frac{1}{2}}-\frac{1}{2} \theta$, P-a.s.. Letting $\delta \downarrow 0$, and then $\varepsilon \downarrow 0$ we deduce $\theta \leq 0$ which induces a contradiction. Therefore,

$$
F_{0}(t, x, 0,0)=\sup _{u \in U} \inf _{v \in V} F(t, x, 0,0, u, v) \geq 0,
$$

and from the definition of $F$, we know that $W$ is a viscosity subsolution of equation (5.1). Finally, the results from the first and the second step prove that $W$ is a viscosity solution of equation (5.1).

Remark 5.3. Similarly, we can prove that $U$ is a viscosity solution of equation (5.2). 


\section{Viscosity Solution of Isaacs' Equation: Uniqueness Theorem}

The objective of this section is to study the uniqueness of the viscosity solution of Isaacs' equation $(5.1)$

$$
\left\{\begin{array}{l}
\frac{\partial}{\partial t} \omega(t, x)+H^{-}\left(t, x, \omega, D \omega, D^{2} \omega\right)=0, \quad(t, x) \in[0, T) \times \mathbb{R}^{n} \\
\omega(T, x)=\Phi(x), \quad x \in \mathbb{R}^{n}
\end{array}\right.
$$

Recall that

$$
H^{-}(t, x, y, p, X)=\sup _{u \in U} \inf _{v \in V}\left\{\frac{1}{2} \operatorname{tr}\left(\sigma \sigma^{T}(t, x, u, v) X\right)+p . b(t, x, u, v)+f(t, x, y, p . \sigma, u, v)\right\},
$$

$t \in[0, T], x \in \mathbb{R}^{n}, y \in \mathbb{R}, p \in \mathbb{R}^{n}, X \in \mathbf{S}^{n}$. The functions $b, \sigma, f$ and $\Phi$ are still supposed to satisfy (H4.1) and (H4.2), respectively.

We will prove the uniqueness for equation (6.1) in the following space of continuous functions $\Theta=\left\{\varphi \in C\left([0, T] \times \mathbb{R}^{n}\right): \exists \widetilde{A}>0\right.$ such that

$$
\left.\lim _{|x| \rightarrow \infty} \varphi(t, x) \exp \left\{-\widetilde{A}\left[\log \left(\left(|x|^{2}+1\right)^{\frac{1}{2}}\right)\right]^{2}\right\}=0 \text {, uniformly in } t \in[0, T]\right\} .
$$

This space of continuous functions endowed with a growth condition which is slightly weaker than the assumption of polynomial growth but more restrictive than that of exponential growth. This growth condition was introduced by Barles, Buckdahn, Pardoux [3] to prove the uniqueness of the viscosity solution of an integro-partial differential equation associated with a decoupled FBSDE with jumps. It was shown in [3] that this kind of growth condition is optimal for the uniqueness and can, in general, not be weakened. We adapt the ideas developed in [3] to Isaacs' equation (6.1) to prove the uniqueness of the viscosity solution in $\Theta$. Since the proof of the uniqueness in $\Theta$ for equation (5.2) is the same we will restrict ourselves only on that of (6.1). Before stating the main result of this section, let us begin with two auxiliary lemmata. Denoting by $K$ a Lipschitz constant of $f(t, x, . .$.$) , that is uniformly in (t, x)$, we have the following

Lemma 6.1. Let $u_{1} \in \Theta$ be a viscosity subsolution and $u_{2} \in \Theta$ be a viscosity supersolution of equation (6.1). Then the function $\omega:=u_{1}-u_{2}$ is a viscosity subsolution of the equation

$$
\left\{\begin{array}{cc}
\frac{\partial}{\partial t} \omega(t, x)+\sup _{u \in U, v \in V}\left\{\frac{1}{2} \operatorname{tr}\left(\sigma \sigma^{T}(t, x, u, v) D^{2} \omega\right)+D \omega \cdot b(t, x, u, v)+K|\omega|+\right. \\
K|D \omega \cdot \sigma(t, x, u, v)|\}=0, & (t, x) \in[0, T) \times \mathbb{R}^{n}, \\
\omega(T, x)=0, \quad x \in \mathbb{R}^{n} . &
\end{array}\right.
$$

The proof of this lemma follows directly that of Lemma 3.7 in [3], it is even simpler because contrary to Lemma 3.7 in [3] we don't have any integral part here in equation (6.1). In analogy to [3] we also have

Lemma 6.2. For any $\widetilde{A}>0$, there exists $C_{1}>0$ such that the function

$$
\chi(t, x)=\exp \left[\left(C_{1}(T-t)+\widetilde{A}\right) \psi(x)\right]
$$

with

$$
\psi(x)=\left[\log \left(\left(|x|^{2}+1\right)^{\frac{1}{2}}\right)+1\right]^{2}, x \in \mathbb{R}^{n},
$$

satisfies

$$
\begin{aligned}
& \frac{\partial}{\partial t} \chi(t, x)+\sup _{u \in U, v \in V}\left\{\frac{1}{2} \operatorname{tr}\left(\sigma \sigma^{T}(t, x, u, v) D^{2} \chi\right)+D \chi \cdot b(t, x, u, v)+K \chi(t, x)+\right. \\
& K|D \chi(t, x) . \sigma(t, x, u, v)|\}<0 \quad \text { in }\left[t_{1}, T\right] \times \mathbb{R}^{n}, \text { where } t_{1}=T-\frac{\widetilde{A}}{C_{1}} .
\end{aligned}
$$


Proof. By direct calculus we first deduce the following estimates for the first and second derivatives of $\psi$ :

$$
|D \psi(x)| \leq \frac{2[\psi(x)]^{\frac{1}{2}}}{\left(|x|^{2}+1\right)^{\frac{1}{2}}} \leq 4, \quad\left|D^{2} \psi(x)\right| \leq \frac{C\left(1+[\psi(x)]^{\frac{1}{2}}\right)}{|x|^{2}+1}, \quad x \in \mathbb{R}^{n} .
$$

These estimates imply that, if $t \in\left[t_{1}, T\right]$,

$$
\begin{aligned}
|D \chi(t, x)| & \leq\left(C_{1}(T-t)+\widetilde{A}\right) \chi(t, x)|D \psi(x)| \\
& \leq C \chi(t, x) \frac{[\psi(x)]^{\frac{1}{2}}}{\left(|x|^{2}+1\right)^{\frac{1}{2}}}
\end{aligned}
$$

and, similarly

$$
\left|D^{2} \chi(t, x)\right| \leq C \chi(t, x) \frac{\psi(x)}{|x|^{2}+1} .
$$

We should notice that the above estimates do not depend on $C_{1}$ because of the definition of $t_{1}$. In virtue with the above estimates we have

$$
\begin{aligned}
& \frac{\partial}{\partial t} \chi(t, x)+\sup _{u \in U, v \in V}\left\{\frac{1}{2} \operatorname{tr}\left(\sigma \sigma^{T}(t, x, u, v) D^{2} \chi\right)+D \chi \cdot b(t, x, u, v)+K \chi(t, x)+\right. \\
& \quad K|D \chi(t, x) \cdot \sigma(t, x, u, v)|\} \\
& \leq-\chi(t, x)\left\{C_{1} \psi(x)-C \psi(x)-C[\psi(x)]^{\frac{1}{2}}-K\right\} \\
& <-\chi(t, x)\left\{C_{1}-[2 C+K]\right\} \psi(x)<0, \text { if } C_{1}>2 C+K \text { large enough. }
\end{aligned}
$$

Now we can prove the uniqueness theorem.

Theorem 6.1. We assume that (H4.1), (H4.2) hold. Let $u_{1}$ (resp., $\left.u_{2}\right) \in \Theta$ be a viscosity subsolution (resp., supersolution) of equation (6.1). Then we have

$$
u_{1}(t, x) \leq u_{2}(t, x), \quad \text { for all }(t, x) \in[0, T] \times \mathbb{R}^{n} .
$$

Proof. Let us put $\omega:=u_{1}-u_{2}$. Then we have, for some $\widetilde{A}>0$,

$$
\lim _{|x| \rightarrow \infty} \omega(t, x) e^{-\widetilde{A}\left[\log \left(\left(|x|^{2}+1\right)^{\frac{1}{2}}\right)\right]^{2}}=0,
$$

uniformly with respect to $t \in[0, T]$. This implies, in particular, that for any $\alpha>0, \omega(t, x)-\alpha \chi(t, x)$ is bounded from above in $\left[t_{1}, T\right] \times \mathbb{R}^{n}$, and that

$$
M:=\max _{\left[t_{1}, T\right] \times \mathbb{R}^{n}}(\omega-\alpha \chi)(t, x) e^{-K(T-t)}
$$

is achieved at some point $\left(t_{0}, x_{0}\right) \in\left[t_{1}, T\right] \times \mathbb{R}^{n}$ (depending on $\alpha$ ). We now have to distinguish between two cases.

For the first case we suppose that: $\omega\left(t_{0}, x_{0}\right) \leq 0$, for any $\alpha>0$.

Then, obviously $M \leq 0$ and $u_{1}(t, x)-u_{2}(t, x) \leq \alpha \chi(t, x)$ in $\left[t_{1}, T\right] \times \mathbb{R}^{n}$. Consequently, letting $\alpha$ tend to zero we obtain

$$
u_{1}(t, x) \leq u_{2}(t, x), \text { for all }(t, x) \in\left[t_{1}, T\right] \times \mathbb{R}^{n} .
$$


For the second case we assume that there exists some $\alpha>0$ such that $\omega\left(t_{0}, x_{0}\right)>0$.

We notice that $\omega(t, x)-\alpha \chi(t, x) \leq\left(\omega\left(t_{0}, x_{0}\right)-\alpha \chi\left(t_{0}, x_{0}\right)\right) e^{-K\left(t-t_{0}\right)}$ in $\left[t_{1}, T\right] \times \mathbb{R}^{n}$. Then, putting

$$
\varphi(t, x)=\alpha \chi(t, x)+(\omega-\alpha \chi)\left(t_{0}, x_{0}\right) e^{-K\left(t-t_{0}\right)}
$$

we get $\omega-\varphi \leq 0=(\omega-\varphi)\left(t_{0}, x_{0}\right)$ in $\left[t_{1}, T\right] \times \mathbb{R}^{n}$. Consequently, since $\omega$ is a viscosity subsolution of (6.2) from Lemma 6.1 we have

$$
\begin{aligned}
& \frac{\partial}{\partial t} \varphi\left(t_{0}, x_{0}\right)+\sup _{u \in U, v \in V}\left\{\frac{1}{2} \operatorname{tr}\left(\sigma \sigma^{T}\left(t_{0}, x_{0}, u, v\right) D^{2} \varphi\left(t_{0}, x_{0}\right)\right)+D \varphi\left(t_{0}, x_{0}\right) . b\left(t_{0}, x_{0}, u, v\right)+\right. \\
& \left.K\left|\varphi\left(t_{0}, x_{0}\right)\right|+K\left|D \varphi\left(t_{0}, x_{0}\right) . \sigma\left(t_{0}, x_{0}, u, v\right)\right|\right\} \geq 0 .
\end{aligned}
$$

Moreover, due to our assumption that $\omega\left(t_{0}, x_{0}\right)>0$ and since $\omega\left(t_{0}, x_{0}\right)=\varphi\left(t_{0}, x_{0}\right)$ we can replace $K\left|\varphi\left(t_{0}, x_{0}\right)\right|$ by $K \varphi\left(t_{0}, x_{0}\right)$ in the above formula. Then, from the definition of $\varphi$ and Lemma 6.2 ,

$$
\begin{aligned}
& 0 \leq \alpha\left\{\frac{\partial \chi}{\partial t}\left(t_{0}, x_{0}\right)+\sup _{u \in U, v \in V}\left\{\frac{1}{2} \operatorname{tr}\left(\sigma \sigma^{T}\left(t_{0}, x_{0}, u, v\right) D^{2} \chi\left(t_{0}, x_{0}\right)\right)+D \chi\left(t_{0}, x_{0}\right) \cdot b\left(t_{0}, x_{0}, u, v\right)+\right.\right. \\
& \left.\left.K \chi\left(t_{0}, x_{0}\right)+K\left|D \chi\left(t_{0}, x_{0}\right) \cdot \sigma\left(t_{0}, x_{0}, u, v\right)\right|\right\}\right\}<0
\end{aligned}
$$

which is a contradiction. Finally, by applying successively the same argument on the interval $\left[t_{2}, t_{1}\right]$ with $t_{2}=\left(t_{1}-\frac{\widetilde{A}}{C_{1}}\right)^{+}$, and then, if $t_{2}>0$, on $\left[t_{3}, t_{2}\right]$ with $t_{3}=\left(t_{2}-\frac{\widetilde{A}}{C_{1}}\right)^{+}$, etc. We get

$$
u_{1}(t, x) \leq u_{2}(t, x), \quad(t, x) \in[0, T] \times \mathbb{R}^{n} .
$$

Thus, the proof is complete.

Remark 6.1. Obviously, since the lower value function $W(t, x)$ is of at most linear growth it belongs to $\Theta$, and so $W(t, x)$ is the unique viscosity solution in $\Theta$ of equation (6.1). Similarly we get that the upper value function $U(t, x)$ is the unique viscosity solution in $\Theta$ of equation (5.2).

Remark 6.2. If the Isaacs' condition holds, that is, if for all $(t, x, y, p, X) \in[0, T] \times \mathbb{R}^{n} \times \mathbb{R} \times$ $\mathbb{R}^{n} \times \mathbf{S}^{n}$

$$
H^{-}(t, x, y, p, X)=H^{+}(t, x, y, p, X),
$$

then the equations (6.1) and (5.2) coincide and from the uniqueness in $\Theta$ of viscosity solution it follows that the lower value function $W(t, x)$ equals to the upper value function $U(t, x)$ which means the associated stochastic differential game has a value.

Remark 6.3. Let us assume that the coefficient of $B S D E(4.5) f(t, x, y, z, u, v) \equiv f(t, x, u, v)$ is independent of $(y, z)$, and denote by $\tilde{W}(t, x)$ (resp., $\tilde{U}(t, x)$ ) the lower value function (resp., the upper value function) defined by Fleming and Souganidis [8], see Remark 4.2. It is shown in [8] that $\tilde{W}(t, x)$ is a viscosity solution in $\Theta$ of (6.1) and $\tilde{U}(t, x)$ a viscosity solution in $\Theta$ of (5.2). Then, due to Theorem 6.1, $W(t, x)=\tilde{W}(t, x)$ and $U(t, x)=\tilde{U}(t, x),(t, x) \in[0, T] \times \mathbb{R}^{n}$. Moreover, if the Isaacs' condition holds then $W(t, x)=\tilde{W}(t, x)=\tilde{U}(t, x)=U(t, x)$.

\section{References}

[1] BAHLALI, K. (2001) Backward stochastic differential equations with locally Lipschitz coefficient. C. R. Acad. Sci., Paris, Sr. I, Math. 333, No.5, 481-486. 
[2] BAHLALI, K., ESSAKY, E.H., HASSANI, M. and PARDOUX, E. (2002) Existence, uniqueness and stability of backward stochastic differential equations with locally monotone coefficient. C. R., Math., Acad. Sci. Paris 335, No.9, 757-762.

[3] BARLES, G., BUCKDAHN, R. and PARDOUX, E. (1997) Backward stochastic differential equations and integral-partial differential equations. Stochastics and Stochastics Reports. Vol.60, 57-83.

[4] BUCKDAHN, R., CARDALIAGUET, P. and RAINER, C. (2004) Nash equilibrium payoffs for nonzero-sum stochastic differential games. SIAM J. Cont. Opt. 43, No.2, 624-642.

[5] CRANDALL, M.G., ISHII, H., LIONS, P.L. (1992) User's guide to viscosity solutions of second order partial differential equations. Bull. Amer. Math. Soc. 27, 1-67.

[6] EL KAROUI, N., PENG, S. and QUENEZ, M.C. (1997) Backward stochastic differential equations in finance. Math. Finance. 7, No.1, 1 - 71.

[7] EVANS, L.C. and SOUGANIDIS, P.E. (1984) Differential games and representation formulas for solutions of Hamilton-Jacobi-Isaacs equations. Indiana Univ. Math. J. 33, 773-797.

[8] FLEMING, W.H. and SOUGANIDIS, P.E. (1989) On the existence of value functions of twoplayer, zero-sum stochastic differential games. Indiana Univ. Math. J. 38, No.2, 293-314.

[9] HAMADENE, S., LEPELTIER, J.P. (1995) Zero-sum stochastic differential games and backward equations. Systems and Control Letters. 24, 259-263.

[10] HAMADENE, S., LEPELTIER, J.P. and PENG, S. (1997) BSDEs with continuous coefficients and stochastic differential games. El Karoui, N. and Mazliak, L. (Eds.), Backward stochastic differential equations. Harlow: Longman. Pitman Res. Notes Math. Ser. 364, 115-128.

[11] HOU, S. and TANG, S. (2006) Switching games of stochastic differential systems. Preprint.

[12] PARDOUX, E. and PENG, S. (1990) Adapted solution of a backward stochastic differential equation. Systems Control Lett. 14, No.1 - 2, 61 - 74.

[13] PARDOUX, E. and PENG, S. (1992) Backward stochastic differential equations and quasilinear parabolic partial differential equations. Stochastic partial differential equations and their applications. Proc. IFIP Int. Conf., Charlotte/NC (USA) 1991, Lect. Notes Control Inf. Sci. $176,200-217$.

[14] PENG, S. (1997) BSDE and stochastic optimizations; Topics in stochastic analysis. YAN, J., PENG, S., FANG, S. and WU, L. Ch.2, Science Press. Beijing (in Chinese).

[15] PENG, S. (1992) A generalized dynamic programming principle and Hamilton-Jacobi-Bellman equation. Stochastics and Stochastics Reports. Vol.38, 119-134.

[16] RAINER, C. (2003) On feedback controls for nonzero-sum stochastic differential games. Preprint. 